\title{
User Pairing, Link Selection and Power Allocation for Cooperative NOMA Hybrid VLC/RF Systems
}

\author{
Mohanad Obeed, Hayssam Dahrouj, Senior Member, IEEE, Anas M. Salhab, Senior Member, IEEE, \\ Salam A. Zummo, Senior Member, IEEE, and Mohamed-Slim Alouini, Fellow, IEEE
}

\begin{abstract}
Despite the promising high-data rate features of visible light communications (VLC), they still suffer from unbalanced services due to blockages and channel fluctuation among users. This paper introduces and evaluates a new transmission scheme which adopts cooperative non-orthogonal multiple access (Co-NOMA) in hybrid VLC/radio-frequency (RF) systems, so as to improve both system sum-rate and fairness. Consider a network consisting of one VLC access point (AP) and multiple strong and weak users, where each weak user is paired with a strong user. Each weak user can be served either directly by the VLC AP, or via the strong user which converts light information received through the VLC link, and forwards the information to the weak user via the RF link. The paper then maximizes a network-wide weighted sum-rate, so as to jointly determine the strong-weak user-pairs, the serving link of each weak user (i.e., either direct VLC or hybrid VLC/RF), and the power of each user message, subject to user connectivity and transmit power constraints. The paper tackles such a mixed-integer non-convex optimization problem using an iterative approach. Simulations show that the proposed scheme significantly improves the VLC network performance (i.e., sum-rate and fairness) as compared to the conventional NOMA scheme.
\end{abstract}

Index Terms-Visible light communication, non-orthogonal multiple-access, cooperative NOMA, energy harvesting, power allocation, weighted sum-rate.

\section{INTRODUCTION}

\section{A. Overview}

Toward meeting the escalating data rates demand, visible light provides a valuable, useful spectrum for transmitting data, which complements conventional radio-frequency (RF) communication systems. As the energy-efficient light emitting diodes (LEDs) have become more popular as light sources in indoor and outdoor environments, visible light communication (VLC) has emerged as a promising energy-efficient solution for transmitting information using LEDs. It has been proven that VLC networks can provide data rates of several Giga-bits per second [1], [2], which makes them a powerful alternative (or complementary) to RF networks. However, the main limitation of VLC systems is that they experience poor fairness

M. Obeed is with the School of Engineering, University of British Columbia, Kelowna V1Y 1V7, BC, Canada (email: mohanad.obeed@ubc.ca).

Hayssam Dahrouj is with the Center of Excellence for NEOM Research, King Abdullah University of Science and Technology (KAUST), Thuwal 23955, Makkah Province, Saudi Arabia (email: hayssam.dahrouj@gmail.com).

A. M. Salhab and S. A. Zummo are with the Department Electrical Engineering, King Fahd University of Petroleum \& Minerals (KFUPM), Dhahran 31261, Saudi Arabia (email: salhab@kfupm.edu.sa, zummo@kfupm.edu.sa).

M.-S. Alouini is with the Department Computer, Electrical, and Mathematical Siences \& Engineering, KAUST, Thuwal 23955, Makkah Province, Saudi Arabia (email: slim.alouini@kaust.edu.sa) and uneven QoS distribution. In other words, in VLC systems, the variance of the the received services at users is high, and so some of the users (hereafter called strong users) would be well covered, while other users (hereafter called weak users) would be poorly covered. This is due to the facts that the VLC channel significantly deteriorates with distance, and that communication through light can be easily blocked by intermediate obstacles. In other words, the communication in VLC networks is also a strong function of the existence of line-ofsight (LoS) links, which in turn get significantly attenuated with distance between the transmitter and the receiver, thereby limiting the coverage area to tiny cells, also known as attocells [3]. The users' field-of-view (FoV) also plays a key role in the individual users' experiences, since increasing the FoV users improves the probability that the $\operatorname{LoS}$ is available but attenuates the received power, while decreasing the FoV does the opposite [4]. This motivates us in this paper to propose and evaluate a new scheme that combines the advantages of cooperative non-orthogonal multiple-access (Co-NOMA), hybrid VLC/RF, and energy harvesting techniques to address the aforementioned VLC design challenges.

In an effort to increase the throughput and improve the fairness in wireless systems, non-orthogonal multiple access (NOMA) technique was introduced in the recent wireless literature. NOMA is based on sharing the resource components (subcarrier, spreading code, or time slot) by more than one user. This can be implemented by assigning a low power for the strong users, and a high power for the weak ones. The weak users then decode their own messages and treat the strong users' messages as interference, while the strong users first decode the weak users' messages, remove it from the total received signal, and finally detect their message. This scheme improves the system performance in VLC networks; however, it cannot extend the system coverage or mitigate the blocking effect.

In RF networks, a recently proposed alternative to NOMA is cooperative NOMA (Co-NOMA), specifically proposed to improve the fairness and strengthen the signal-to-noise ratio (SNR) at the weak users [5], by exploiting any redundant information in NOMA. This could be implemented in practical networks, where the strong user can also work as a relay to assist the weak user. The weak user then combines both signals coming from the transmitter and from the the strong user. This technique (Co-NOMA) has not been applied in VLC networks in the past literature, mainly because of the physical challenges incurred while forwarding the light signal by the strong user to the weak user. One possible technique to 
overcome such challenges, however, is to convert the received light signal at the strong user into an RF signal, and then forward it to the weak user through an RF link. This paper adopts such Co-NOMA scheme in an hybrid VLC/RF system, and thoroughly illustrates its capabilities in enhancing the sum-rate and fairness as compared to the conventional NOMA scheme.

Harvesting the energy is also considered in this paper, where the strong user might not be satisfied to forward the weak user's signal using its own power. Hence, the received visible light at the strong user can be used to charge a power storage and this can be used as a power source to forward the weak user's signal. The energy can be harvested in VLC networks using the received direct current (DC) without affecting the power of the transmitted signal [6], [7].

Consider a VLC network consisting of one AP and two predefined sets of strong and weak users. Each weak user can be paired by one strong user through an RF link. The strong user is served directly by the VLC link. Each weak user can be either served by the VLC link, or by the strong user through the RF link by means of Co-NOMA. The performance of the system becomes, therefore, a function of the user pairing, link selection and the messages powers. The paper then focuses on maximizing a network-wide weighted sum-rate, so as to jointly pair the strong and weak users, allocate their respective powers, and select the mode of operation of each weak user (i.e., direct VLC or hybrid VLC/RF).

\section{B. Related Work}

This subsection presents an overview of the recent stateof-art in VLC systems, with a special focus on the works which mitigate SNR fluctuations, manage NOMA networks, and analyse CO-NOMA in the realm of VLC systems.

Different techniques have been investigated in the literature to mitigate the SNR fluctuations so as to increase the system coverage probability, and improve the system performance in terms of both total achievable data rate and system fairness [8]-[17]. Among the common solutions to strike such a trade-off between throughput and fairness is deploying hybrid VLC/RF networks [9]-[13], coordinating transmissions [8], [14]-[17], and supporting the network with relay-assisted VLC transmission [18], [19]. Hybrid VLC/RF technology, in particular, helps supporting the VLC standalone systems by one or multiple RF APs. The main idea in such systems is to compromise between the high VLC capacity and high $\mathrm{RF}$ coverage, which is often realized by assigning the users which suffer from interference, blockages, frequent handover, or low-quality channel in VLC network to be served by RF $\mathrm{AP}(\mathrm{s})$, while serving the rest of users by the VLC AP(s) [4]. In the same direction, the authors in [8] tackle the problem of jointly optimizing the time slots and assigning the APs to the users in a hybrid VLC/WiFi system. Supplementing VLC by RF AP(s) is further shown to support mobility and decrease the handover overhead [9], [10]. Authors in [11] show that the users that experience blockages with high rate should be served by the RF network. References [12], [13] allocate the power and assign the users to VLC and RF APs to reduce the interference effect and to maximize the system capacity and fairness. References [8]-[13], however, do not consider neither non-orthogonal multiple access schemes and do not allow any level of cooperation among users, unlike our current paper which addresses the rate-fairness balancing problem by means of adopting a Co-NOMA scheme in hybrid VLC/RF systems.

To further alleviate the VLC limitations, cooperation among APs is proposed in the recent literature, e.g., [8], [14], [15], by merging the cells and boosting the users quality-ofservice (QoS) by coordinating the APs transmissions. Such coordination allows the APs to cancel the interference [14][16], increase the cell coverage [8], and mitigate the blockages effect [17]. Relaying is also investigated in VLC networks to extend the VLC coverage [18]-[20]. In [18] and [19], dualhop hybrid VLC/RF links are proposed to serve uncovered users by means of relaying. Under such schemes, the relay harvests the energy and receives the signal through the first hop VLC link, and then forwards the signal to the receiver through the second hop RF link. To harvest the energy in VLC networks, the authors of [21], [22] present and study the concept of simultaneous lightwave information and power transfer (SLIPT) and focus on designing the receiver that realizes SLIPT. The authors of [7] extend the concept of SLIPT to be used in MISO-multiuser VLC system.

NOMA has extensively been investigated in RF networks, and has shown to enhance the spectral efficiency and the system fairness [23], [24]. In the context of VLC networks, reference [25] shows the superiority of NOMA over orthogonalfrequency division multiple access (OFDMA) with regard to sum-rate. The authors in [26] evaluate the NOMA-VLC system and compare it to orthogonal multiple-access (OMA)VLC scheme with and without QoS constraints. The authors in [27] evaluate and compare the NOMA and OMA when the locations and the vertical orientations of the devices is changing. For multiple APs, the work in [28] proposes a gain ratio power allocation (GRPA) method and compares it with the fixed power allocation method, when the users' movement model follows the random walk model. For multi-cell VLC networks, the users in [29] are classified based on the received interference. In reference [29], special resource blocks are assigned for the interfering users, while NOMA is applied for the interference-free users. The authors of [30] consider the problem of optimizing the beamforming vectors through minimizing the transmit power subject to QoS constraints in NOMA-MISO multi-user dynamic and static systems. The authors in [31] extend the work of [30] for multi-cell system model and develop centralized and distributed interference management schemes for VLC systems. Recently, the authors in [32] investigate the outage capacity for a limited system model, consisting of one VLC AP and two users (one is covered, while the other is out of the coverage). The covered user uses the Co-NOMA scheme to forward the uncovered user signal through the RF link, and the uncovered user does not have the option to be served directly through the VLC link. The authors of [33] derive closed-form expressions for the outage capacity and the sum throughput of cooperative NOMA hybrid VLC/RF two-user system. Unlike our work, 
the authors of [32], [33] do not consider the problem of optimizing the power, user pairing and the link selection. Besides, reference [33] does not examine the impact of using hybrid VLC/RF and cooperative NOMA in mitigating the blockage effect and extending the coverage.

In all the above studies, however, jointly allocating the power, selecting the link, and pairing the users have not been studied in the context of CO-NOMA in hybrid VLC/RF systems, which the current paper tackles.

\section{Contributions}

Unlike the aforementioned papers, this paper introduces and evaluates a cooperation scheme among users in VLC networks based on hybrid VLC/RF Co-NOMA with simultaneous information and power transfer. More precisely, the paper considers a system model, consisting of one VLC AP and multiple users classified as either strong or weak users, strong and weak users. A transmission scheme based on hybrid VLC/RF CoNOMA scheme is proposed, where the strong users receive their own signals through the direct VLC link. The paper transmission scheme then provides two options for each weak user, either to be served by the direct VLC link, or to be served by the relayed hybrid VLC/RF link that is provided by a strong user which can help the weak user by forwarding their signal through the RF link. This technique extends the VLC coverage area and helps serving the blocked users, which leads to improving the fairness and balancing the load in VLC systems. To this end, the paper addresses the problem of maximizing a network-wide weighted sum-rate, so as to jointly pair the strong and weak users, allocate their respective powers, and select the link of operation of every weak user (i.e., direct VLC or hybrid VLC/RF). The paper contributions can then be summarized as follows:

- The paper proposes system model that allows cooperation among users using RF links, harvests the power using the transmitted DC bias, and uses the NOMA principle to improve the system performance. We propose combining these techniques in VLC networks to extend the coverage area, reach the blocked users, and improve the received data rates at the weak users without using extra power compared to the non-cooperative NOMA system.

- The paper formulates a mixed discrete-continuous optimization problem that jointly pairs the users, selects the serving link for each weak user, and allocates the messages' power to maximize the weighted sum-rate of the system under user connectivity and maximum power constraints.

- The paper solves the mixed-integer non-convex optimization problem by proposing an iterative algorithm, which iteratively finds each of the optimization parameter by fixing all others. The paper particularly derives closedform solutions for the power allocation problem, and proposes well-chosen heuristics for finding the user pairing and link selection parameters. The paper also proposes updating the weights of the weighted-sum rate objective in an outer loop to achieve a proportional fairness of the system where, in each iteration, the weight of every user is determined to be inversely proportional to the longterm average rate of that user [34].

- The paper compares the proposed system model to the the conventional NOMA-VLC scheme and the proposed algorithms to a simpler baseline approach. The paper simulation results illustrate how the proposed hybrid VLC/RF Co-NOMA scheme significantly improves the VLC network performance, both in terms of sum-rate and fairness, as compared to the conventional NOMA scheme.

Finally, the following points show how the proposed scheme is different from Co-NOMA proposed in RF networks: Firstly, in RF systems, the weak users are able to combine the $\mathrm{RF}$ signals from the direct and relayed links using combination techniques (e.g., maximal ratio combining). In VLC/RF systems, however, the weak user should select either to be served through direct or relayed links. Hence, in RF systems, any combination technique is physically tangible, while in VLC/RF system, a selection technique is rather required. Secondly, the power transfer in our scheme is implemented by exploiting the transmitted DC-bias, while in RF schemes, a power splitting or time switching is used for simultaneous power and information transfer. Thirdly, because of the peak power constraint in VLC systems, the sum rate expression is mathematically different from that of RF. The transmit power is further constrained according to the input current, which must be within the linear operational range of LEDs. Fourthly, in RF systems, the strong user must use either half duplex (which degrades the capacity) or full duplex (which causes self interference) schemes, so as to act as a relay. In our proposed scheme, however, the strong user can receive and transmit at the same time, without being subject to self-interference. This is because the VLC links innately do not interfere with RF links.

The rest of this paper is organized as follows. The system and channel models are presented and discussed in Section II. Section III formulates the optimization problem and presents the proposed algorithms. Simulation results are illustrated in Section IV. Finally, we conclude the paper in Section V.

\section{System And Channel Models}

\section{A. System Model}

The system model considered in this paper consists of a VLC AP and $N_{u}=2 K$ multiple users, where $K$ is the number of pairs. The users are classified into two sets, a set $\mathbb{U}_{s}$ of $K$ users, defined as strong users, which are the users with strong VLC channels, and a set $\mathbb{U}_{w}$ of $K$ weak users, which are the users with the weak VLC channels. Fig. 1 shows an example of the considered network which serves three weak users, and three strong users. Without loss of generality, the paper assumes that the number of users are even, which facilitates the investigation of the proposed CoNOMA technique. In the case where the number of users are odd, one can assume the existence of one more additional virtual user with a zero channel gain (and equivalently a zero achievable rate). 


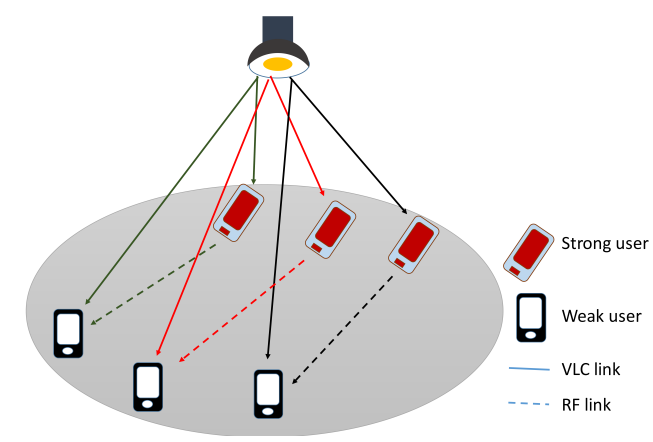

Fig. 1. An example of the considered system with three weak users, and three strong users.

The paper adopts a Co-NOMA scheme, where the available bandwidth is divided equally into $K$ blocks. Each spectrum block is shared by a pair of users (one strong and one weak user). The weak user in each pair can be served either directly by the VLC AP through the VLC link, or by the dual-hop hybrid VLC/RF link through the paired strong user. In the case where the weak user is not well served by the VLC, the strong user would act as an energy harvesting relay which harvests the energy from the VLC AP (using the received visible light), and would then use it to forward the message to the paired weak user using the RF link. Hereinafter, we illustrate the VLC and RF channel model, the energy harvesting signals, and the transmission scheme.

\section{B. VLC and RF Channel Model}

According to [35], the VLC channel between the AP and the $j$ th user, denoted by $h_{j}$, is given by

$$
h_{j}=\frac{(m+1) A_{p}}{2 \pi d_{j}^{2}} \cos ^{m}(\phi) g_{o f} \cos (\theta) f(\theta),
$$

where $A_{p}$ is the photo-detector (PD) physical area, $m=$ $-\left(\log _{2}\left(\cos \left(\theta_{\frac{1}{2}}\right)\right)^{-1}\right.$ is the Lambertian index, $\theta_{\frac{1}{2}}$ is the semiangle of half power, $d_{j}$ is the distance between the AP and the $j$ th user, $g_{o f}$ is the gain of the optical filter, $\phi$ is the LED radiance angle, $\theta$ is the PD incidence angle, and $f(\theta)$ is the gain of the optical concentrator given by

$$
f(\theta)= \begin{cases}\frac{n^{2}}{\sin ^{2}(\Theta)}, & \theta \leq \Theta \\ 0, & \theta>\Theta\end{cases}
$$

where $n$ is the refractive index and $\Theta$ is the semi-angle of the user's field-of-view (FoV). The considered system model does not assume that there are reflecting objects or walls, which is aligned with the majority of the literature when the system model consists of only one VLC transmitter. Hence, we assume that if the LoS between the AP and the PD, the channel is zero, otherwise the channel is given by (1).

According to [36], the RF channel gain in indoor environment between the $j$ th user and the $i$ th user is given by

$$
G_{i, j}^{R F}=\left|H_{i, j}^{(R F)}\right|^{2} 10^{-\frac{L\left(d_{i, j}\right)}{10}},
$$

where $H_{i, j}^{(R F)}$ is the RF multipath propagation channel and $L\left(d_{i, j}\right)$ is the path loss between the $j$ th user and the $i$ th user, where $d_{i, j}$ is the distance between $i$ and $j$ users.

\section{Transmission Scheme: Direct Links}

In the rest of this paper, we associate the subscript $i$ with weak users, and $j$ with strong users. Following the NOMA principle, suppose that one weak user $i$ is paired with one strong user $j$, the expression of the transmitted signal from the AP towards the user-pair $i$ and $j$ is given by

$$
y_{i, j}=\nu \sqrt{P_{j}^{(s)}} s_{j}+\nu \sqrt{P_{i}^{(w)}} s_{i}+\nu b,
$$

where $P_{j}^{(s)}$ and $P_{i}^{(w)}$ are the powers of the strong and weak users assigned for $s_{j}$ and $s_{i}$ messages, respectively, $b$ is the direct-current (DC) that must be added to guarantee that the transmitted signal is non-negative, and $\nu$ is the proportionality factor of the electric to optical power conversion. The received signals at the $j$ th and $i$ th users, respectively, are given by

$$
\begin{aligned}
& y_{j}=\nu \rho h_{j}^{(s)}\left(\sqrt{P_{j}^{(s)}} s_{j}+\sqrt{P_{i}^{(w)}} s_{i}\right)+\nu \rho h_{j}^{(s)} b+n_{j}, \\
& y_{i}=\nu \rho h_{i}^{(w)}\left(\sqrt{P_{j}^{(s)}} s_{j}+\sqrt{P_{i}^{(w)}} s_{i}\right)+\nu \rho h_{i}^{(w)} b+n_{i},
\end{aligned}
$$

where $h_{j}^{(s)}$ and $h_{i}^{(w)}$ are the channels of the $j$ th strong user and the $i$ th weak user, respectively, $\rho$ is the detector responsivity, and $n_{j}$ or $n_{i}$ are the noise components which can be modeled as real zero-mean additive white Gaussian noise variable (AWGN) with variance $\sigma^{2}=N_{v} B_{v}$, where $N_{v}$ is the noise power spectral density (PSD) and $B_{v}$ is the modulation bandwidth. In this paper, we consider only the additive Gaussian noise without considering the shot noise [37]. The received DC part $\rho \nu h_{j}^{(s)} b$ at the strong user can be separated by a capacitor and directed to the energy harvesting circuit [6], while at the weak user, the DC part $\rho \nu h_{i}^{(w)} b$ can be removed using a capacitor. The strong user detects the weak user's message $s_{i}$ and then removes it or directs it to the weak user, while the weak user decodes his own signal $s_{i}$ by treating the strong user's signal $s_{j}$ as interference. Hence, the achievable data rate of the strong user signal is upper bounded by (equation (27) in [38])

$$
R_{j}^{(s)}\left(P_{j}^{(s)}\right)=\frac{B_{v}}{2 K} \log _{2}\left(1+\frac{c \nu^{2} \rho^{2} h_{j}^{(s) 2} P_{j}^{(s)}}{B_{v} N_{v} / K}\right) .
$$

The achievable data rate of the weak user data decoded by the strong user is upper bounded by (equation (28) in [38])

$$
R_{j \rightarrow i}^{(w)}\left(P_{i}^{(w)}, P_{j}^{(s)}\right)=\frac{B_{v}}{2 K} \log _{2}\left(1+\frac{c \nu^{2} \rho^{2} h_{j}^{(s) 2} P_{i}^{(w)}}{B_{v} N_{v} / K+c \nu^{2} \rho^{2} h_{j}^{(s) 2} P_{j}^{(s)}}\right) .
$$

The achievable data rate received at the weak user from the VLC direct link (DL) is upper bounded by (equation (28) in [38])

$$
R_{i, D L}^{(w)}\left(P_{i}^{(w)}, P_{j}^{(s)}\right)=\frac{B_{v}}{2 K} \log _{2}\left(1+\frac{c \nu^{2} \rho^{2} h_{i}^{(w) 2} P_{i}^{(w)}}{B_{v} N_{v} / K+c \nu^{2} \rho^{2} h_{i}^{(w) 2} P_{j}^{(s)}}\right),
$$

where $c=\min \left\{\frac{1}{2 \pi e}, \frac{e b^{2}}{2 I_{H}^{2} \pi}\right\}$, where $e$ is the Euler number and $I_{H}$ is the maximum limit of the deriving input current to LEDs. Since $b$ is equal to $\frac{I_{H}}{2}, c=\frac{1}{2 \pi e}$. 


\section{Transmission Scheme: Relayed Links and Energy Harvest-} ing

The paper assumes that the strong user can work also as a relay that has the ability to harvest the energy from the light intensity, and then to utilize it to forward the decoded weak user's signal. To harvest the energy, a capacitor separates the DC component from the received electrical signal and forwards it to the energy harvesting circuit [6], [7], [21]. The received energy at the $j$ th user can be expressed as [39]

$$
E_{j}=f \rho \nu V_{t} h_{j} b \ln \left(1+\frac{\rho h_{j} \nu b}{I_{0}}\right) .
$$

where $V_{t}$ is the thermal voltage, $f$ is the fill factor, and $I_{0}$ is the dark saturation current of the PD. Suppose that the amplitude of the transmitted signal is $A$. The DC-bias and the signal amplitude $A+b$ must, therefore, be within the maximum and minimum input currents to make sure that the LED transmitter is operating in the linear region. In other words, let $I_{H}$ and $I_{L}$ be the maximum and minimum limits of the input currents for the LEDs that guarantee a linear output optical power. The constraint $A \leq \min \left(b-I_{L}, I_{H}-b\right)$ must then be achieved. The deriving power to the LED is related to $A$ and $b$ by $P_{\max }=(A-b)^{2}$. This means that the maximum allowed deriving power $P_{\max }$ at the AP is a decreasing function of the DC-bias $b$. The DC-bias can be optimized to balance between the received harvested energy and the transmit power, but this is out of the scope of this paper. Hence, the DC-bias at the AP is assumed to be fixed and is given by $b=\frac{I_{H}+I_{L}}{2}$ which maximizes the total transmit power [7]. Therefore, the maximum allowed deriving power is given by

$$
P_{\text {max }}=\left(\frac{I_{H}-I_{L}}{2}\right)^{2} .
$$

The strong user is assumed to be able to receive the light signal and transmit the RF signal at the same time. However, the energy storage device cannot charge and discharge at the same time (i.e., the receiver cannot harvest the energy and transmit the data at the same time). Hence, suppose that $T_{1}$ is the time spent to charge the battery and $T_{2}$ is the time used to discharge or re-transmit data through the RF link. Therefore, the RF re-transmission power is given by $P_{j, R F}=\frac{E_{j} T_{1}}{T_{2}}$. Under the assumption that $T_{1}=T_{2}$, the achievable data rate of the weak user that can be offered by the strong user $j$ through the RF link is given by

$$
R_{i, j}^{R F}=\frac{B_{f, j}}{2} \log \left(1+\frac{G_{i, j}^{R F} P_{j, R F}}{B_{f, j} N_{R F}}\right),
$$

where $B_{f, j}$ is the RF modulation bandwidth at the user $j$ and $N_{R F}$ is the PSD of the RF signal. If the number of weak users that are served through relayed VLC/RF links is more than one, the RF bandwidth must be divided between weak users; otherwise, additional interference terms would arise. This paper assigns orthogonal RF bandwidth for each pair connected through the RF link equally, so as to nullify the wireless interference among the active RF links. Suppose that the number of weak users that are served through RF links is $N_{f}$ and the total modulation bandwidth available for RF transmission is $B_{f}$, the modulation bandwidth at user $j$ is $B_{f, j}=\frac{B_{f}}{N_{f}}$.

From (12) and (8), the achievable data rate at the weak user through the hybrid relayed link (RL) can be expressed as

$$
R_{i, R L}^{(w)}\left(P_{i}^{(w)}, P_{j}^{(s)}\right)=\min \left(R_{i, j}^{R F}, R_{j \rightarrow i}^{(w)}\left(P_{i}^{(w)}, P_{j}^{(s)}\right)\right) .
$$

\section{Problem Formulation and Solutions}

Our goal in this paper is to maximize the weighted sum of the achievable data rates under user connectivity and transmit power constraints. To formulate and tackle the problem, we should answer the three interlinked questions: 1) How should the users be paired? 2) What is the serving link of each weak user (i.e., direct VLC link or relayed VLC/RF link)? 3) How should the total power at the AP be allocated among users? This section formulates these questions as an optimization problem to jointly obtain the power allocation vector $\mathbf{P}$, the pairing index matrix $\mathbf{Z}$, and the link selection index vector $\mathrm{x}$ to maximize the network-wide weighted sum-rate. To simplify the paper presentation and avoid the confusion of the notations of the data rates, we introduce Table I which defines the different achievable data rates, their symbols, and expressions. It can be seen that increasing the DC-bias leads to increasing the harvested energy at the strong users, but also decreasing the total transmit power (power assigned for information) coming form the direct VLC links. However, numerically, we find that optimizing the DC-bias is negligible, in terms of improving the sum rate. This is because the effect of increasing the DC-bias on the total transmit power is dominating, which makes the effect of changing the DC-bias on the harvested energy is approximately absent. Therefore, this paper does not adopt the problem of optimizing the DCbias.

Define the user pairing $K \times K$ matrix $\mathbf{Z}$, where the entries of $\mathbf{Z}$ are defined as follows:

$$
z_{i, j}= \begin{cases}1, & \text { if weak user } i \text { is paired with strong user } j, \\ 0, & \text { otherwise }\end{cases}
$$

Define the link selection indicator vector $\mathbf{x}$, where the entries of $\mathbf{x}$ are defined as follows:

$x_{i}= \begin{cases}1, & \text { if user } i \text { is served through the RF/VLC link, } \\ 0, & \text { if user } i \text { is served through the direct VLC link. }\end{cases}$

From (15), the number of weak users that are connected through RF link is given by $N_{f}=\sum_{i=1}^{K} x_{i}$. The rate in (12) becomes, therefore, a function of $\mathbf{x}$ as shown in Table I. The summation of the achievable data rate of the $i$ th weak user and the $j$ th strong user is given by

$$
\begin{aligned}
& R_{i, j}\left(P_{i}^{(w)}, P_{j}^{(s)}, z_{i, j}, \mathbf{x}\right)=z_{i, j} R_{j}^{(s)}\left(P_{j}^{(s)}\right)+z_{i, j} \\
& \left(1-x_{i}\right) R_{i, D L}^{(w)}\left(P_{i}^{(w)}, P_{j}^{(s)}\right)+z_{i, j} x_{i} R_{i, R L}^{(w)}\left(P_{i}^{(w)}, P_{j}^{(s)}, \mathbf{x}\right) .
\end{aligned}
$$


TABLE I

DATA RATES NOTATIONS

\begin{tabular}{|l|l|l|}
\hline Symbol & Definition & Expression \\
\hline$R_{j}^{(s)}\left(P_{j}^{(s)}\right)$ & $\begin{array}{l}\text { The achievable data rate of the } j \text { th strong } \\
\text { user signal }\end{array}$ & $R_{j}^{(s)}\left(P_{j}^{(s)}\right)=\frac{B_{v}}{2 K} \log _{2}\left(1+\frac{c \nu^{2} \rho^{2} h_{j}^{(s) 2} P_{j}^{(s)}}{B_{v} N_{v} / K}\right)$ \\
\hline$R_{j \rightarrow i}^{(w)}\left(P_{i}^{(w)}, P_{j}^{(s)}\right)$ & $\begin{array}{l}\text { The achievable data rate of the weak user } \\
\text { data decoded by the strong user }\end{array}$ & $R_{j \rightarrow i}^{(w)}\left(P_{i}^{(w)}, P_{j}^{(s)}\right)=\frac{B_{v}}{2 K} \log _{2}\left(1+\frac{c \nu^{2} \rho^{2} h_{j}^{(s) 2} P_{i}^{(w)}}{B_{v} N_{v} / K+c \nu^{2} \rho^{2} h_{j}^{(s) 2} P_{j}^{(s)}}\right)$ \\
\hline$R_{i, D L}^{(w)}\left(P_{i}^{(w)}, P_{j}^{(s)}\right)$ & $\begin{array}{l}\text { The achievable data rate received at the weak } \\
\text { user from the VLC direct link (DL) }\end{array}$ & $R_{i, D L}^{(w)}\left(P_{i}^{(w)}, P_{j}^{(s)}\right)=\frac{B_{v}}{2 K} \log _{2}\left(1+\frac{c \nu^{2} \rho^{2} h_{i}^{(w) 2} P_{i}^{(w)}}{B_{v} N_{v} / K+c \nu^{2} \rho^{2} h_{i}^{(w) 2} P_{j}^{(s)}}\right)$ \\
\hline$R_{i, j}^{R F}(\mathbf{x})$ & $\begin{array}{l}\text { The achievable data rate of the } i \text { th weak user } \\
\text { offered by the } j \text { th strong user through the RF } \\
\text { link }\end{array}$ & $R_{i, j}^{R F}(\mathbf{x})=\frac{B_{f}}{2 \sum_{x_{i}}^{K} x_{i}} \log \left(1+\frac{G_{i, j}^{R F} P_{j, R F} R}{B_{f} N_{R F} / \sum_{i}^{K} x_{i}}\right)$. \\
\hline$R_{i, R L}^{(w)}\left(P_{i}^{(w)}, P_{j}^{(s)}, \mathbf{x}\right)$ & $\begin{array}{l}\text { The achievable data rate at the weak user } \\
\text { through the hybrid relayed link (RL) }\end{array}$ & $R_{i, R L}^{(w)}\left(P_{i}^{(w)}, P_{j}^{(s)}\right)=\min \left(R_{i, j}^{R F}(\mathbf{x}), R_{j \rightarrow i}^{(w)}\left(P_{i}^{(w)}, P_{j}^{(s)}\right)\right)$ \\
\hline$R_{i, j}\left(P_{i}^{(w)}, P_{j}^{(s)}, z_{i, j}, \mathbf{x}\right)$ & $\begin{array}{l}\text { The summation of the achievable data rate } \\
\text { of the } i \text { th weak user and } j \text { th strong user }\end{array}$ & $\begin{array}{l}R_{i, j}\left(P_{i}^{(w)}, P_{j}^{(s)}, z_{i, j}, \mathbf{x}\right) \\
\left.x_{i}\right) R_{i, D L}^{(w)}\left(P_{i}^{(w)}, P_{j}^{(s)}\right)+z_{i, j} x_{i} R_{i, R L}^{(w)}\left(P_{i}^{(w)}, P_{j}^{(s)}, \mathbf{x}\right)\end{array}$ \\
\hline
\end{tabular}

The weighted sum-rate of the system users is given by

$$
\begin{aligned}
& \sum_{i=1}^{K} \sum_{j=1}^{K} R_{i, j}\left(P_{i}^{(w)}, P_{j}^{(s)}, z_{i, j}, x_{i}, w_{i}^{(w)}, w_{j}^{(s)}\right)= \\
& \sum_{i=1}^{K} \sum_{j=1}^{K} w_{j}^{(s)} z_{i, j} R_{j}^{(s)}\left(P_{j}^{(s)}\right)+w_{i} z_{i, j}\left(1-x_{i}\right) R_{i, D L}^{(w)}\left(P_{i}^{(w)}, P_{j}^{(s)}\right) \\
& \quad+w_{i}^{(w)} z_{i, j} x_{i} \min \left(R_{i, j}^{R F}(\mathbf{x}), R_{j \rightarrow i}^{(w)}\left(P_{i}^{(w)}, P_{j}^{(s)}\right)\right), \quad(17)
\end{aligned}
$$

where the weights $w_{i}^{(w)}$ and $w_{j}^{(s)}(\forall i, j)$ are imposed to balance between the system sum-rate and the system fairness. Based on expression (17), the considered optimization problem can now be expressed mathematically as follows:

$$
\begin{aligned}
\max _{\mathbf{P}, \mathbf{z}, \mathbf{x}} & \sum_{i=1}^{K} \sum_{j=1}^{K} R_{i, j}\left(P_{i}^{(w)}, P_{j}^{(s)}, z_{i, j}, x_{i, j}, w_{i}^{(w)}, w_{j}^{(s)}\right)(18 \mathrm{a}) \\
\text { s.t. } & \sum_{i=1}^{K} \sum_{j=1}^{K} z_{i, j}\left(P_{i}^{(w)}+P_{j}^{(s)}\right) \leq P_{\max } \\
& \sum_{j=1}^{K} z_{i, j}=1, \forall i, \sum_{i=1}^{K} z_{i, j}=1, \forall j, \\
& z_{i, j} \in\{0,1\}, \forall i, j \\
& x_{i} \in\{0,1\}, \forall i \\
& 0 \leq P_{j}^{(s)} \leq P_{i}^{(w)} \forall i, j,
\end{aligned}
$$

where the optimization is over the continuous power variable $\mathbf{P}$, and the discrete association variables $\mathbf{z}$ and $\mathbf{x}$. Constraint (18b) is imposed for the maximum transmit power. Constraints in (18c) guarantee that each strong user is paired only with one weak user. From constraints (18b) and (18c), it can be shown that constraint (18b) can be equivalently written as $\sum_{i=1}^{K} P_{i}^{(w)}+\sum_{j=1}^{K} P_{j}^{(s)} \leq P_{\max }$. Constraint (18d) imposes that the weak user receives the information either from the direct VLC link or from the relayed hybrid VLC/RF link. Constraint $(18 \mathrm{e})$ is the power constraint that is imposed for successive interference cancellation in NOMA system.

The problem in (18) is a challenging mixed non-convex combinatorial optimization problem. The paper tackles such a difficult problem through a heuristic approach. The main idea is to solve the problem for each parameter when the other parameters are fixed. Specifically, for the power allocation problem, we find closed-form solutions for the formulated non-convex optimization problem. For the user pairing problem, we use the Hungarian method for strong-to-weak users assignment problem. For the link selection problem, we find the optimal solution by first generating a $K \times K$ matrix that reduces the number of candidate vectors from $2^{K}$ to $K$. The overall algorithm then iterates among the above three steps so as to jointly determine the user pairing, link selection, and power allocation. Although such solution does not lead to the optimal solution of problem (18), the simulations section of the paper illustrates how the proposed solution notably improves the performance of VLC network in terms of sumrate and fairness.

\section{A. Power Allocation}

This section solves the optimization problem (18) when the $\mathbf{Z}$ matrix and $\mathbf{x}$ vector are fixed. In particular, we find the power allocation for fixed user pairing and link selection. Under the given $\mathbf{Z}$ and $\mathbf{x}$, the weighted sum maximization problem can be formulated as follows

$$
\begin{aligned}
\max _{\mathbf{P}} & \sum_{i=1}^{K} \sum_{j=1}^{K} R_{i, j}\left(P_{i}^{(w)}, P_{j}^{(s)}, z_{i, j}, x_{i, j}, w_{i}^{(w)}, w_{j}^{(s)}\right)( \\
\text { s.t. } & \sum_{i=1}^{K} \sum_{j=1}^{K} z_{i, j}\left(P_{i}^{(w)}+P_{j}^{(s)}\right) \leq P_{\max } \\
& 0 \leq P_{j}^{(w)} \leq P_{i}^{(s)} \forall i, j .
\end{aligned}
$$

Clearly, problem (19) is not easy to be solved because of the presence of interference terms in the objective function. However, in the following, we provide closed-form solutions for the power allocation. The idea of the proposed solution is that we use a substitution method to solve this problem. We first introduce auxiliary variables $q_{i, j}, i=1, \ldots, K, j=$ $1, \ldots, K$. We then find optimal closed-form solutions of the powers $P_{j}^{s}$ and $P_{i}^{w}$ in terms of these auxiliary variables. 
Then, we substitute these solutions in the original problem to find optimal solutions of these auxiliary variables. In other words, we define a variable $q_{i, j}$ as the power budget of a pair consisting of the $i$ th weak user and $j$ th strong user, i.e., $q_{i, j}=z_{i, j}\left(P_{i}^{(w)}+P_{j}^{(s)}\right)$. Then, we can solve problem (19) by first breaking it into $K$ sub-problems to find $P_{i}^{(w)}$ and $P_{j}^{(s)},(\forall i, j)$, the solution of which depends on one main problem which solves the power budgets $q_{i, j}, \forall i, j$. Hence, for the $k$ th problem, suppose that the weak user $i$ is paired with the strong user $j$ (i.e., $z_{i, j}=1$ ). We then distinguish between two cases, i.e., either the weak user is served by the hybrid VLC/RF link (called Case 1 in the rest of the paper), or the weak user is served by the VLC link (called Case 2 in the rest of the paper).

1) Case 1: Users $i$ and $j$ are paired, and the weak user $i$ is served through the hybrid VLC/RF link. For a given $q_{i, j}$, the problem of finding $P_{i}^{(w)}$ and $P_{j}^{(s)}$ in this case can be formulated as follows

$$
\begin{array}{cc}
\max _{P_{i}^{(w)}, P_{j}^{(s)}} & w_{j}^{(s)} R_{j}^{(s)}\left(P_{j}^{(s)}\right)+w_{i}^{(w)} \min \left(R_{i, j}^{R F}(\mathbf{x}),\right. \\
& \left.R_{j \rightarrow i}^{(w)}\left(P_{i}^{(w)}, P_{j}^{(s)}\right)\right) \\
\text { s.t. } & P_{i}^{(w)}+P_{j}^{(s)}=q_{i, j} \\
& 0 \leq P_{j}^{(s)} \leq P_{i}^{(w)} .
\end{array}
$$

To solve problem (20), note first that the problem above must be carefully treated because of the min term in the objective function. Also, for a fixed $\mathbf{x}$, observe that the function $R_{i, j}^{R F}(\mathbf{x})$ is fixed, as it is not a function of the power variables $P_{i}^{(w)}$ and $P_{j}^{(s)}$.

Remark 1. In problem (20), the resulting optimal value of $R_{j \rightarrow i}^{(w)}\left(P_{i}^{(w)}, P_{j}^{(s)}\right)$ must be less than or equal to the resulting optimal value of $R_{i, j}^{R F}(\mathbf{x})$. To prove that, we can see that increasing $P_{i}^{(w)}$ (decreasing $P_{j}^{(s)}$ ) increases $R_{j \rightarrow i}^{(w)}\left(P_{i}^{(w)}, P_{j}^{(s)}\right.$ ) and decreases the achievable data rate of the strong user at the same time. This means that increasing $P_{i}^{(w)}$ to have $R_{j \rightarrow i}^{(w)}\left(P_{i}^{(w)}, P_{j}^{(s)}\right)$ larger than $R_{i, j}^{R F}(\mathbf{x})$ would fix the data rate of the weak user at $R_{i, j}^{R F}(\mathbf{x})$ and would decrease the data rate of the strong user. In other words, if $\hat{P}_{i}$ is the value that would make $R_{i, j}^{R F}(\mathbf{x})=R_{j \rightarrow i}^{(w)}\left(\hat{P}_{i}, P_{j}^{(s)}\right)$, the optimal $P_{i}^{(w)}$ must be less than or equal to $\hat{P}_{i}$.

Based on Remark 1, problem (20) can be expressed equivalently as follows

$$
\begin{array}{cll}
\max _{P_{i}^{(w)}, P_{j}^{(s)}} & w_{j}^{(s)} R_{j}^{(s)}\left(P_{j}^{(s)}\right)+w_{i}^{(w)} R_{j \rightarrow i}^{(w)}\left(P_{i}^{(w)}, P_{j}^{(s)}\right) & (21 \mathrm{a}) \\
\text { s.t. } & P_{i}^{(w)}+P_{j}^{(s)}=q_{i, j}, & \text { (21b) } \\
& 0 \leq P_{j}^{(s)} \leq P_{i}^{(w)}, & \text { (21c) } \\
& R_{j \rightarrow i}^{(w)}\left(P_{i}^{(w)}, P_{j}^{(s)}\right) \leq R_{i, j}^{R F}(\mathbf{x}) .
\end{array}
$$

Proposition 1. The optimal solution of problem (21) is given by

$$
P_{j}^{(s)}=\min \left(\eta_{i, j, 1}, \eta_{i, j, 2}\right)
$$

and

$$
P_{i}^{(w)}=q_{i, j}-P_{j}^{(s)}
$$

where $\eta_{i, j, 1}=\frac{-1+\sqrt{1+q_{i, j} \Psi_{j}^{(s)}}}{\Psi_{j}^{(s)}}, \eta_{i, j, 2}=\frac{q_{i, j} \Psi_{j}^{(s)}+1-A}{A \Psi_{j}^{(s)}}, \Psi_{j}^{(s)}=$ $\frac{c \nu^{2} \rho^{2} h_{j}^{(s) 2}}{B_{v} N_{v} / K}, \Psi_{i}^{(w)}=\frac{c \nu^{2} \rho^{2} h_{i}^{(w) 2}}{B_{v} N_{v} / K}$, and $A=2^{2 R_{i, j}^{R F}(\mathbf{x}) K / B_{v}}$.

Proof. See Appendix A.

2) Case 2: Users $i$ and $j$ are paired, and the weak user $i$ is served through the direct VLC link. The problem in this case can be formulated as

$$
\begin{array}{cl}
\max _{P_{i}^{(w)}, P_{j}^{(s)}} & w_{j}^{(s)} R_{j}^{(s)}\left(P_{j}^{(s)}\right)+w_{i}^{(w)} R_{i, D L}^{(w)}\left(P_{i}^{(w)}, P_{j}^{(s)}\right) \\
\text { s.t. } & P_{i}^{(w)}+P_{j}^{(s)}=q_{i, j} \\
& 0 \leq P_{j}^{(s)} \leq P_{i}^{(w)}
\end{array}
$$

Because of the interference term in $R_{i, D L}^{(w)}\left(P_{i}^{(w)}, P_{j}^{(s)}\right)$, the optimization problem (24) is still nonconvex. However, the authors of [40] (Section IV) tackle a problem of similar structure and show that the optimal solution has a closedform. By setting the derivative of the objective function of (24) equal to zero, we obtain

$$
\begin{aligned}
& \frac{d}{d P_{j}^{(s)}}\left[w_{j}^{(s)} R_{j}^{(s)}\left(P_{j}^{(s)}\right)+w_{i}^{(w)} R_{i, D L}^{(w)}\left(P_{i}^{(w)}, P_{j}^{(s)}\right)\right] \\
& =\frac{w_{j}^{(s)} B_{v}}{2 K\left(1 / \Psi_{j}^{(s)}+P_{j}^{(s)}\right)}-\frac{w_{i}^{(w)} B_{v}}{2 K\left(1 / \Psi_{i}^{(w)}+P_{j}^{(s)}\right)}=0
\end{aligned}
$$

One can readily verify that the second derivative of the objective function is always negative if $\Psi_{j}^{(s)} \geq \Psi_{i}^{(w)}$ and $w_{i}^{(w)} / w_{j}^{(s)}<\Psi_{j}^{(s)} / \Psi_{i}^{(w)}$, i.e., the objective function is concave in such case. Therefore, the optimal solution can be obtained by solving equation (25), which leads to a unique root $P_{j}^{(s)}=\Omega_{i, j}$, where $\Omega_{i, j}$ is given by

$$
\Omega_{i, j}=\frac{w_{i} \Psi_{i}^{(w)}-w_{j}^{(s)} \Psi_{j}^{(s)}}{\Psi_{j}^{(s)} \Psi_{i}^{(w)}\left(w_{j}^{(s)}-w_{i}^{(w)}\right)},
$$

where the conditions that $w_{i}^{(w)} / w_{j}^{(s)}<\Psi_{j}^{(s)} / \Psi_{i}^{(w)}$ and $q_{i, j}>$ $2 \Omega_{i, j}$ must be satisfied, and $P_{i}^{(w)}=q_{i, j}-\Omega_{i, j}$.

3) Determination of $q_{i, j}$ : The previous analysis of both Case 1 and Case 2 allows to determine the powers $P_{j}^{(s)}$ and $P_{i}^{(w)}$ as a function of $q_{i, j}$, i.e., equations (22) and (26). By substituting the corresponding expressions of $P_{j}^{(s)}$ and $P_{i}^{(w)}$ in problem (19), one can formulate the following optimization problem:

$$
\begin{aligned}
\max _{q_{i, j}} & \sum_{i=1}^{K} \sum_{j=1}^{K} x_{i} z_{i, j}\left(w_{j}^{(s)} F_{j}^{(s)}\left(q_{i, j}\right)+w_{i}^{(w)} F_{i}^{(w)}\left(q_{i, j}\right)\right) \\
& +\sum_{i=1}^{K} \sum_{j=1}^{K}\left(1-x_{i}\right) z_{i, j} w_{i}^{(w)} \frac{B_{v}}{2 K} \log _{2}\left(1+\Omega_{i, j} \Psi_{j}^{(s)}\right) \\
& +\sum_{i=1}^{K} \sum_{j=1}^{K} \frac{\left(1-x_{i}\right) z_{i, j} w_{i}^{(w)} B_{v}}{2 K} \log _{2}\left(\frac{q_{i, j} \Psi_{i}^{(w)}+1}{\Omega_{i, j} \Psi_{i}^{(w)}+1}\right)(27 \mathrm{a}) \\
\text { s.t. } \quad & \sum_{i=1}^{K} \sum_{j=1}^{K} q_{i, j}=P_{\max }, \\
& q_{i, j} \geq 0, \forall i, j,
\end{aligned}
$$


where $F_{j}^{(s)}\left(q_{i, j}\right)$ and $F_{i}^{(w)}\left(q_{i, j}\right)$ can be written either as

$$
F_{j}^{(s)}\left(q_{i, j}\right)=R_{j}^{(s)}\left(\eta_{i, j, 1}\right)=\frac{B_{v}}{2 K} \log _{2}\left(\sqrt{\Psi_{j}^{(s)} q_{i, j}+1}\right)
$$

and

$$
F_{i}^{(w)}\left(q_{i, j}\right)=R_{i, R L}^{(w)}\left(\eta_{i, j, 1}\right)=\frac{B_{v}}{2 K} \log _{2}\left(\sqrt{\Psi_{j}^{(s)} q_{i, j}+1}\right)
$$

or as

$$
F_{j}^{(s)}\left(q_{i, j}\right)=R_{j}^{(s)}\left(\eta_{i, j, 2}\right)=\frac{B_{v}}{2 K} \log _{2}\left(\frac{\Psi_{j}^{(s)} q_{i, j}+1}{A}\right)
$$

and

$$
F_{i}^{(w)}\left(q_{i, j}\right)=R_{i, R L}^{(w)}\left(\eta_{i, j, 2}\right)=R_{i, j}^{R F}(\mathbf{x}) .
$$

At this stage, we cannot decide what are the exact expressions of the functions $F_{j}^{(s)}\left(q_{i, j}\right)$ and $F_{i}^{(w)}\left(q_{i, j}\right), \forall i, j$, because both functions depend on the solution of (22) (i.e., whether the optimal power allocation of the strong user is given by $\eta_{i, j, 1}$ or by $\left.\eta_{i, j, 2}\right)$. Since $q_{i, j}$ is still unknown, we next derive a closed-form solution for $q_{i, j}$, for both possible expressions of $F_{j}^{(s)}\left(q_{i, j}\right)$ and $F_{i}^{(w)}\left(q_{i, j}\right)$.

Proposition 2. The optimal solution of problem (27) is expressed as

$$
q_{i, j}=\left[\frac{w B_{v}}{2 K \lambda}-\frac{1}{\Psi_{j}^{(s)}}\right]^{+},
$$

where $w=w_{i}^{(w)}$ if $x_{i}=0, w=w_{j}^{(s)}$ if $x_{i}=1,[n]^{+}$ means $\max (0, n)$, and $\lambda$ is the dual variable related to the total transmitting power constraint $(27 b)$ and can be found by substituting (32) in constraint (27b).

Proof. See Appendix B.

The steps of the power allocation algorithm are presented in Algorithm 1 below, which explains how to best allocate the powers of the users (for fixed users' pairing and link selection).

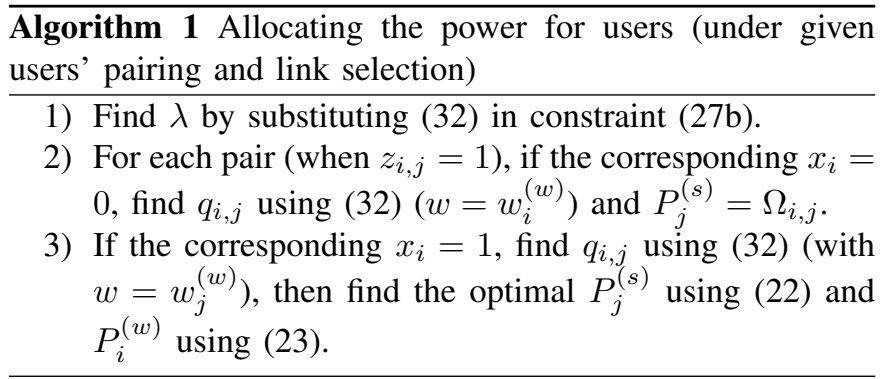

\section{B. Updating the Weights}

To best balance the users rates across the network, this paper chooses to update the weights using the classical proportional fairness approach, e.g., see [34] and references therein. We first give each user initial weights and solve the problem for the powers. From the resulted powers, we calculate the achievable rate of each user and update the weights using equations $w_{i}^{(w)}=\frac{1}{R_{i}^{(w)}} \forall i$ and $w_{j}^{(s)}=\frac{1}{R_{j}^{(s)}} \forall j$.
At the iteration $k$, we update the weights using equations $w_{i}^{(w)}=\frac{1}{\bar{R}_{i}^{(w)}}$ and $w_{j}^{(s)}=\frac{1}{\bar{R}_{j}^{(s)}}$, where $\bar{R}_{i}{ }^{(w)}$ and $\bar{R}_{j}{ }^{(s)}$ are the long term average rates resulted from all iterations of the $i$ th weak user and $j$ th strong user, respectively [34]. There is one condition in selecting the weights that is it is not reasonable that the weight of the strong user is higher than the weight of the paired weak user, since this deteriorates the overall fairness and also gives non-positive power if we use the derived closed-forms. Hence, through the outer loop iterations, if $\bar{R}_{j}$ is dropped below $\bar{R}_{i}$, we select $w_{j}^{(s)}=\alpha w_{i}^{(w)}$, where $\alpha \lesssim 1$, i.e., $\alpha$ is strictly less than 1 (yet sufficiently close to $1)$.

\section{User Pairing Optimization}

Section III-A determines the power allocation for given user pairing and link selection. In this section, we provide how to pair the users under a given fixed power allocation and link selection. For the given fixed power allocation and fixed link selection, the optimization problem (18) can be formulated as

$$
\begin{aligned}
& \max _{\mathbf{Z}} \sum_{i=1}^{K} \sum_{j=1}^{K} w_{j}^{(s)} z_{i, j} R_{j}^{(s)}\left(P_{j}^{(s)}\right) \\
& +w_{i}^{(w)} z_{i, j}\left(1-x_{i}\right) R_{i, D L}^{(w)}\left(P_{i}^{(w)}, P_{j}^{(s)}\right) \\
& +w_{i}^{(w)} z_{i, j} x_{i} \min \left(R_{i, j}^{R F}(\mathbf{x}), R_{j \rightarrow i}^{(w)}\left(P_{i}^{(w)}, P_{j}^{(s)}\right)\right)(33 \mathrm{a}) \\
& \text { s.t. } \quad \sum_{j=1}^{K} z_{i, j}=1, \forall i \\
& \sum_{i=1}^{K} z_{i, j}=1, \forall j \\
& z_{i, j} \in\{0,1\}, \forall i, j
\end{aligned}
$$

The above optimization problem is a integer linear programming, which simply pairs each of the weak users to one (and only one) strong user based on the utility values. Such problem is considered as a one-to-one linear assignment problem [41], where it can be solved using one of the conventional matching algorithms, e.g., the Hungarian method [41]. It is important to note that the Hungarian method provides the optimal solution if the problem is one-to-one assignment [42], which is the case of problem (33).

\section{Link Selection Optimization}

This section now focuses on the link selection problem for fixed power allocation and user pairing, which are determined in the previous subsections. The problem of selecting the optimal links for the weak users, i.e., either to be served by the VLC AP, or via the strong user which relays the information from the VLC AP to the weak user through the hybrid VLC/RF links (even for given power allocation and user pairing) is not easy to be tackled. This problem in general is NP-hard. More precisely, the link selection vector determines which link should serve each user and also affects the capacity of RF links since, as shown in Table $\mathrm{I}, R_{i, j}^{R F}(\mathbf{x})$ is a function of $\sum_{i=1}^{K} x_{i}$. This means that each user cannot decide which 
link is better for it, because this decision depends on the other users' decisions, which makes the problem quite hard to tackle. To find the optimal link selection vector, we need to try all the $2^{K}$ candidates vectors and select the best one. In order to reduce the algorithmic complexity of our proposed approach, we introduce a heuristic method that first generates a $K \times K$ matrix that reduces the number of candidates vector from $2^{K}$ to $K$ vectors. In particular, define a matrix $S$, where the first row in $S$ hosts the rates of the weak users coming from the relayed links subtracted form the rates coming from the direct link when $\sum_{i=1}^{K} x_{i}=1$. Similarly, the second row in $S$ hosts the rates of the weak users coming from the relayed links subtracted form the rates coming from the direct link when $\sum_{i=1}^{K} x_{i}=2$. Construct all other rows of the matrix $S$ in a similar fashion, i.e., the $K$ th row hosts the rates of the weak users coming from the relayed links subtracted form the rates coming from the direct link, where $\sum_{i=1}^{K} x_{i}=K$.

Select afterwards the vector $\mathbf{x}$ corresponding to the highest values of each row. More specifically, consider row $k$ of matrix $S$. The highest $k$ values of the row are then set to 1 ; the other entries are set to 0 . This results in having $K$ different $\mathrm{x}$ vectors, out of which we select the one that maximizes the objective weighted sum function. Such method has a polynomial computational complexity $(O(K \times K+K))$, which is significantly simpler than the complexity of the exhaustive search, i.e., $O\left(2^{K}\right)$. In addition, the proposed approach guarantees the optimal solution because it finds the optimal $\mathbf{x}$ vector for each case of $\sum_{i=1}^{K} x_{i}$ and tries all of them to select the maximizing one.

\section{E. Overall Algorithm}

In this section, we propose a joint solution for problem (18) that considers the power allocation, the link selection, and the user pairing problems jointly. We wish to mention that, in the literature, there are different solutions proposed to solve mixed integer non-convex problems from a holistic perspective. For example, mixed integer non-linear problems can be tackled using graph-theory based techniques [43], simulated annealing [44], particle swarm intelligence [45], genetic algorithms [44]- [45], and evaluation strategies [44]. Such approaches, however, are computationally exhaustive, and oftentimes suffer from numerical scalability issues, i.e., they may not be suitable for the reasonably sized networks assumed in our paper. Therefore, we propose an iterative solution that gets benefit from solving each variable on its own while fixing the other two variables. Since each of the continuous and discrete variables of problem (18) are determined separately, the paper proposes solving (18) using an iterative algorithm that finds the three variables in a cyclic way. In particular, we first initialize the user pairing and link selection, and find the powers using the proposed closed-form solutions based on the given user pairing and link selection initial values. Afterwards, we find the $\mathbf{Z}$ matrix based on the found allocated power and link selection. The link selection vector is finally updated to maximize the weighted sum objective function. These steps are repeated until convergence. To guarantee that Algorithm 2 converges, we check the objective function (18a) at each step. If the current value is smaller than the previous one, the current solution is ignored and the previous solution is adopted. This means that at each iteration, the objective function (18a) either increases or stays fixed. Since the objective function is bounded from above, the proposed algorithm is guaranteed to converge. The steps of the overall solution are given in Algorithm 2 Table.

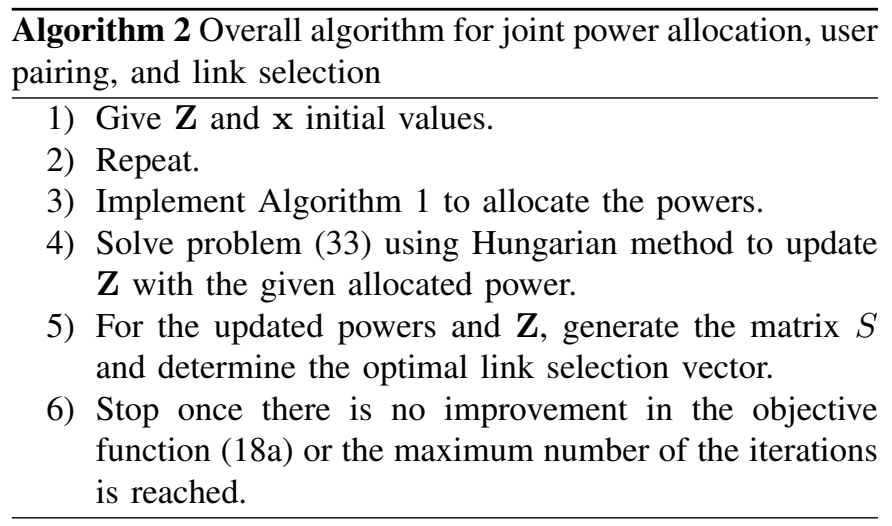

It is important to note that Algorithm 2 is a suboptimal solution and does not guarantee global or local optimal solution due to the fact that the formulated problem is NPhard and not jointly convex. However, the proposed solution is powerful and feasible because of several reasons. First, It provides the optimal solution of any variable if we fix the others. Second, the proposed solution provides closed-form solutions for the power allocation. Such analytical expressions are particularly useful for finding the global optimal solution when the size the problem is small. In addition, since the constraints are separable, the solution of the proposed alternating optimization method guarantees to achieve the constraints at each step. Finally, the proposed algorithm converges in a fast way as shown in Fig. 9. The figure shows that the overall algorithm converges in approximately 4 iterations.

\section{F. Computational Complexity}

To best characterize the computational complexity of the proposed algorithm, we note first that the overall algorithm, i.e., Algorithm 2 solves three distinct problems sequentially. The problem of power allocation can be solved by implementing the derived closed-forms. In particular, $3 K$ equations are needed to be solved to find the variables $P_{j} \forall j, P_{i} \forall i$, and $q_{i, j} \forall z_{i, j}=1$. For the user pairing problem, the computational complexity of Hungarian method is in the order of $O\left(K^{3}\right)$ [46]. Finally, as shown earlier, the link selection problem has a polynomial computational complexity $O(K \times K+K)$.

\section{G. Baseline Approaches}

1) Baseline 1 (NOMA approach): To quantify the contribution of proposed system model, we adopt NOMA approach as a baseline approach. The difference between NOMA and the proposed Co-NOMA scheme is that Co-NOMA allows the strong users to forward the weak users' signals through RF links (i.e., there is cooperation among users), which provides two options for the weak users, either to be served by the VLC 
TABLE II

SiMULATION PARAMETERS

\begin{tabular}{|l|l|}
\hline Parameter Name & $\begin{array}{l}\text { Parameter } \\
\text { Value }\end{array}$ \\
\hline Bandwidth of VLC AP, $B$ & $20 \mathrm{MHz}$ \\
The physical area of PDs, $A_{p}$ & $1 \mathrm{~cm}^{2}$ \\
Half-intensity radiation angle, $\theta_{1 / 2}$ & $60^{\circ}$ \\
Gain of optical filter, $g_{o f}$ & 1 \\
Refractive index, $n$ & 1.5 \\
Optical-to-electrical conversion factor, & $0.58[\mathrm{~A} / \mathrm{W}]$ \\
$\rho$ & \\
Noise PSD of LiFi, $N_{0}$ & $10^{-21} \mathrm{~A}^{2} / \mathrm{Hz}$ \\
Maximum input bias current, $I_{H}$ & $800 \mathrm{~mA}$ \\
Minimum input bias current, $I_{L}$ & $400 \mathrm{~mA}$ \\
Fill factor, $f$ & 0.75 \\
Electric-to-optical conversion factor, $\nu$ & $15 \mathrm{~W} / \mathrm{A}$ \\
Thermal voltage, $V_{t}$ & $25 \mathrm{mV}$ \\
Dark saturation current of the PD, $I_{0}$ & $10^{-10} \mathrm{~A}$ \\
LED height, & $3 \mathrm{~m}$ \\
User height & 0.85 \\
\hline RF & \\
\hline The breakpoint distance & $16 \mathrm{mHz}$ \\
Bandwidth & $2.4 \mathrm{GHz}$ \\
Central carrier frequency & $45^{\circ}$ \\
Angle of arrival/departure of LoS & $3 \mathrm{~dB}$ \\
Shadow fading standard deviation (be- & \\
fore the breakpoint) & $5 \mathrm{~dB}$ \\
Shadow fading standard deviation (after & \\
the breakpoint) & $-174 \mathrm{dBm} / \mathrm{Hz}$ \\
PSD of the noise &
\end{tabular}

AP or by the paired strong user through the hybrid VLC/RF link. In NOMA, however, the weak users have only one option, which is to be served through the direct VLC link (i.e., there is no cooperation among users in NOMA). NOMA can, therefore, be seen as a special case of the formulated problem, where the link selection vector $\mathbf{x}$ is set to zero throughout the optimization problem. Therefore, the optimal NOMA scheme can be found by allocating the power using the closed-form solutions (26) and (32) for all possible user pairings. Under such approach, however, uncovered and blocked users would not be served. It is important to note that if a strong user $j$ is paired with a blocked or uncovered user $i$ (i.e., $\Psi_{i}^{(w)}=0$ ), the power allocation for this pair is distributed as $P_{j}^{(s)}=q_{i, j}$, where $q_{i, j}$ is given by $q_{i, j}=\frac{w_{j}^{(s)} B_{v}}{2 K \lambda}-\frac{1}{\Psi_{j}^{(s)}}$, which can be proven in a similar fashion as in proposition 2 .

2) Baseline 2: For the sake of additional algorithmic comparison, we also provide a simple solution from optimization perspective. Specifically, for pairing the users, we propose that the best strong user is paired with the worst weak one, the second best strong user is paired with the second worst weak user, and so on. The rationale behind adopting such approach as a benchmarking baseline is its capability to provide a relative fairness at a low computational complexity. For the link selection vector, this baseline chooses that each blocked or uncovered user (i.e., the users which have zero VLC channel) must be served through the relayed VLC/RF link, while the remaining weak users must be served through the direct VLC link. Under the above user pairing and link selection simple strategies, the power is then allocated using the derived closed-form solutions found in section III-A.

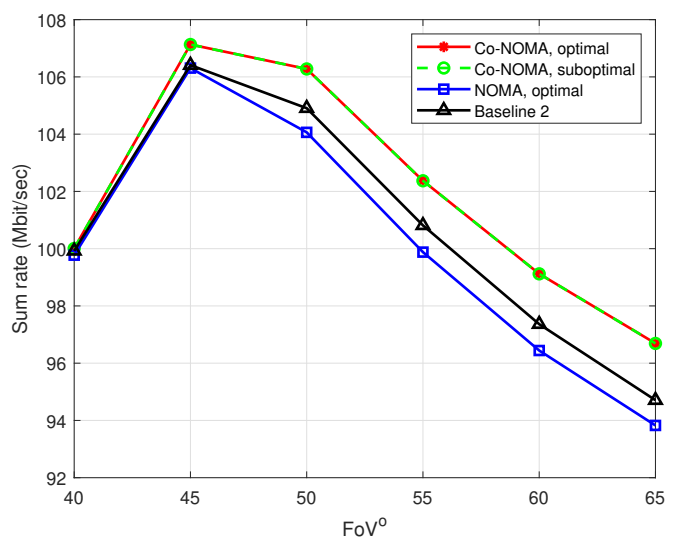

Fig. 2. Sum-rate versus users' FoV when number of users is 6 , the cell radius is $2.5 \mathrm{~m}$, and the blockage rate 0.1 .

\section{Simulation Results}

This section evaluates the performance of the proposed hybrid VLC/RF Co-NOMA scheme and the proposed solutions in terms of both sum-rate and system fairness. We investigate the FoV effects, number of users, blockage rate, and the cell size. Simulation parameters are given in Table II. The performance of the proposed algorithms is assessed through Monte-Carlo simulations, where every point in the numerical results is the average of implementing 1000 different user distributions within the given cell. The blockage rate is defined as the number of times that the user is blocked over the times of total simulation realizations. We use Jain's fairness index to measure the system fairness, which is given by $\frac{\left(\sum_{j=1}^{K} \sum_{i=1}^{K} R_{i, j}\right)^{2}}{2 K\left(\sum_{j=1}^{K} \sum_{i=1}^{K} R_{i, j}^{2}\right)}$.

Fig. 2 compares the proposed hybrid VLC/RF Co-NOMA scheme and the exhaustive search with NOMA and the baseline approaches by plotting the sum-rate against the users' FoV. It can be seen from the figure that increasing the FoV of users leads to increasing the sum-rate, and then decreasing it for all approaches. Such behavior is due to the fact that the small users' FoV provides a potential for having some users to be uncovered, or to have have zero LoS channel gains. As the users' FoV first increases, the probability of coverage increases, which increases the sum-rate. But after some point, increasing the FoV would affect the channel quality as illustrated through equations (1) and (2), which explains why the sum-rate decreases for larger values of the users' FoV. Most importantly, the figure shows how the proposed Co-NOMA scheme outperforms all other algorithms for all values of the FoV, which highlights the important role of the proposed scheme in increasing the network throughput as compared to the classical NOMA scheme.

On the other hand, Fig. 3 compares the proposed hybrid VLC/RF Co-NOMA scheme versus both NOMA and the proposed baseline approaches. Fig. 3 plots Jain's fairness index versus the users' FoV. It can be seen that the fairness is low when the users' FoV is low, since the users which are far from AP would be out of the view (i.e., the LoS channel is zero), while the users that are close to the AP 


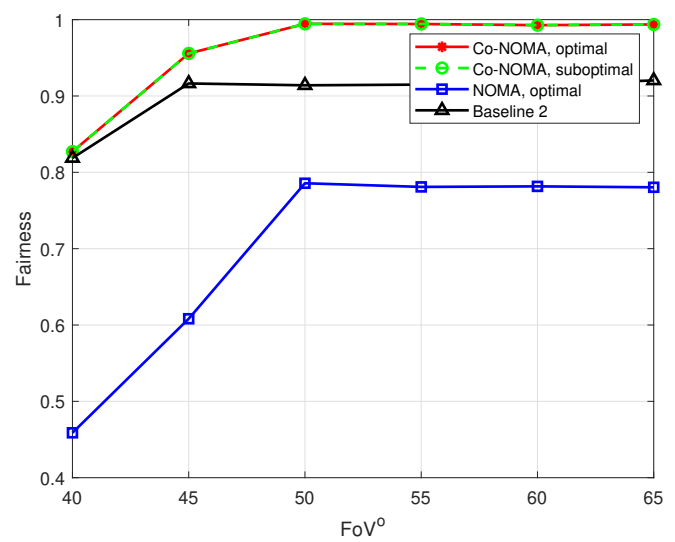

Fig. 3. System fairness versus users' FoV when number of users is 6 , the cell radius is $2.5 \mathrm{~m}$, and the blockage rate 0.1 .

would get a good quality of service because of their channel quality. As the users' FoV increases, the probability that the number of covered users increases within a fixed certain area. Fig. 3 particularly illustrates how the fairness of the proposed Co-NOMA scheme outperforms both NOMA scheme and the proposed baseline approach for all values of the FoV. In fact, both Fig. 2 and Fig. 3 suggest that the proposed hybrid VLC/RF Co-NOMA scheme (optimal or suboptimal) outperforms NOMA and the proposed baseline 2 in terms of both fairness and sum-rate. The improvement in terms of fairness is particularly pronounced, because NOMA scheme cannot reach the out-of-coverage or blocked users, while the proposed hybrid VLC/RF Co-NOMA scheme can reach them through the hybrid VLC/RF relayed link. In addition, the hybrid VLC/RF links can provide the maximum fairness (rather than the direct VLC link) among the strong and weak users without affecting the sum-rate, as also illustrated earlier in Section III-A, Case 1.

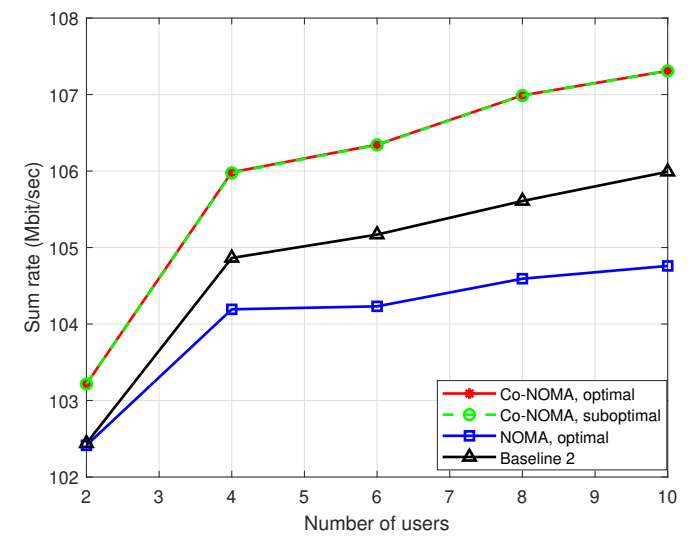

Fig. 4. Sum-rate versus number of users in the system when the cell radius is $2.5 \mathrm{~m}$, the blockage rate 0.1 , and the user $\mathrm{FoV}=50^{\circ}$.

Fig. 4 plots the sum-rate versus the total number of users located within a cell of radius $2.5 \mathrm{~m}$, while Fig. 5 plots the fairness of the same users and with the same cell size. In general, increasing the number of users in the system increases

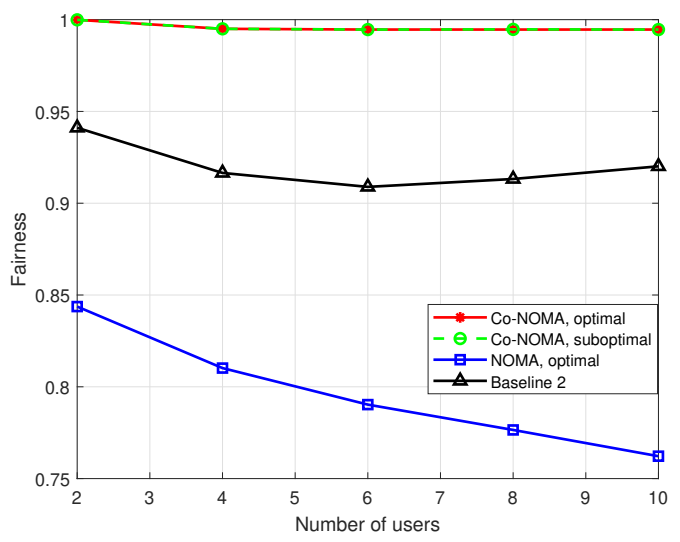

Fig. 5. System fairness versus number of users in the system when the cell radius is $2.5 \mathrm{~m}$, the blockage rate 0.1 , and the user $\mathrm{FoV}=50^{\circ}$.

the sum-rate, but decreases the system fairness. However, this decrease in fairness (in Fig. 5) is significant in the NOMA scheme and negligible in the proposed hybrid VLC/RF CoNOMA scheme. On the other hand, the sum-rate in the CoNOMA scheme increases in a faster rate than in NOMA. Figs. 4 and 5 also show that the exhaustive search approach and the proposed Co-NOMA scheme almost provide the same performance, which is much better than the proposed baseline approaches.

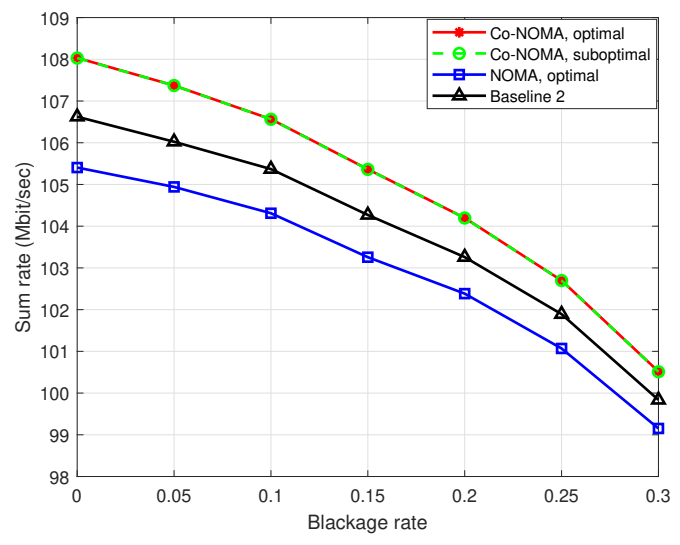

Fig. 6. Sum-rate versus blockage rate when, $N_{u}=6$, the cell radius is 2.5 $\mathrm{m}$, and the users' $\mathrm{FoV}=50^{\circ}$.

Fig. 6 shows the effect of the blockage rate on the sumrate. Increasing the blockage rate decreases the probability of the availability of the VLC LoS links to the users. In other words, the number of blocked users increases, which leads to decreasing the sum-rate of the system. Fig. 6 particularly illustrates how the proposed hybrid VLC/RF Co-NOMA is better than NOMA for all given blockage rates, even when there is no blockage at all. This is because of the selection diversity at the weak user in Co-NOMA (the weak user in CoNOMA can select the link that provides a maximum rate), while the weak user in NOMA has only one option to be served through (i.e., the VLC link).

The effect of the blockage rate on the fairness is shown 


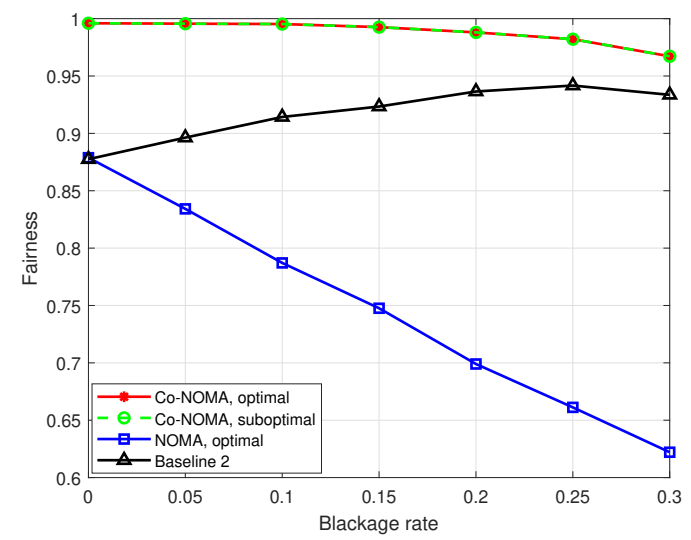

Fig. 7. System fairness versus blockage rate when, $N_{u}=6$, the cell radius is $2.5 \mathrm{~m}$, and the users' $\mathrm{FoV}=50^{\circ}$.

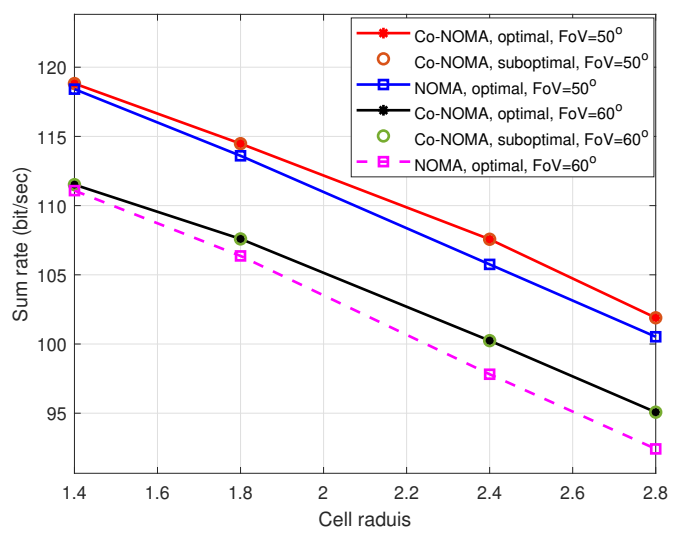

Fig. 8. Sum-rate versus the cell size when, $N_{u}=6$, blockage rate is 0.1 , and with different users' FoV.

in Fig. 7. The figure shows that increasing the blockage rate has a small impact on the fairness of the proposed hybrid VLC/RF Co-NOMA scheme until some point. This is because all the blocked users are considered as weak users and could be served through the paired strong users using the relayed link. But increasing the blockage rate further may result in having the number of blocked users greater than half of the total number of users, which affects also the fairness of the proposed Co-NOMA, albeit to a lesser degree than the impact shown on the NOMA fairness performance. The fairness of the baseline 2 increases with blocking rate since when there is no blockage, since all the users in the baseline 2 approach are served through the direct link, which results in fairness that is similar to NOMA. As the blockage rate increases, the number of served users through the relayed link increases, which results in approaching the maximum fairness reached by the proposed Co-NOMA scheme.

Fig. 8 and Fig. 9 show the impact of increasing the cell size on the sum-rate and fairness, respectively. As the cell size increases, the average channel quality decreases and the probability of having uncovered users increases. As a result, the sum-rate decreases as the cell size increases for CoNOMA and NOMA and with different users' FoV.

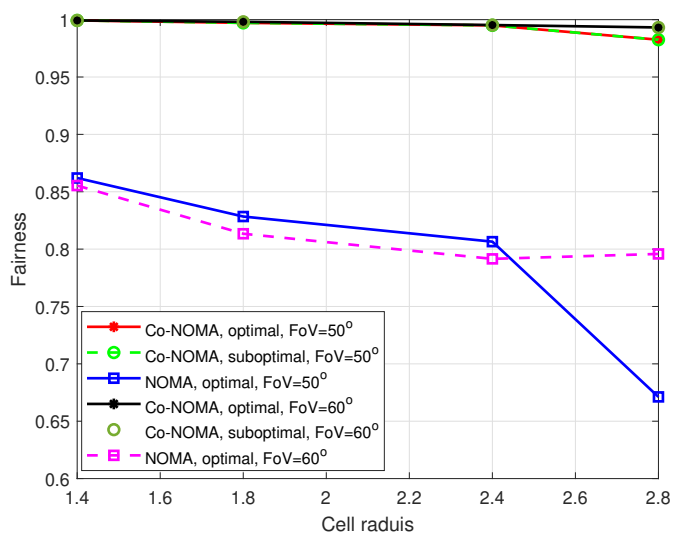

Fig. 9. System fairness versus the cell size when, $N_{u}=6$, blockage rate is 0.1 , and with different users' FoV.

On the other hand, Fig. 9 shows that the fairness slightly decreases as the cell-radius increases for the proposed hybrid VLC/RF Co-NOMA scheme. This is because such scheme extends the coverage area by the RF link and increases the probability of coverage. In contrast, the NOMA scheme cannot reach the out-of-coverage users, which leads to having a high rate of reduction in system fairness. However, increasing the users' FoV would increase the coverage probability, but at the expense of decreasing the channel quality; thereby degrading the system sum-rate as shown in Fig. 8. Once again, Fig. 8 and Fig. 9 highlight how the proposed Co-NOMA scheme outperforms the NOMA approach, both in terms of sum-rate and fairness, and for different cell sizes.

From all the above figures, we see that the proposed scheme provides a significant improvement in fairness over NOMA scheme. In particular, Figures 3, 5, 7, and 9 show such improvement with a gain that ranges between $12 \%$ to $54 \%$, and this improvement increases with increasing the number of users, the blockage rate, or the required coverage area. In general, figures 3, 5, 7, and 9 demonstrate that the proposed scheme empowers VLC networks to be scalable with increasing the number of users, the blockage rate, and the required coverage area.

The proposed scheme and algorithms also improve the sum-rate compared to the non-cooperative NOMA scheme as shown in Figures 2, 4, 6, and 8. The reason why the amount of improvement is only around 3\% in the sum rate plot case is due to the fact that NOMA scheme tends to allocate more power for the strong users to achieve the optimal solution. In other words, for example, if the system consists of two users, one is close to the AP and the other is blocked or out-of-coverage (i.e., the channel is zero), NOMA scheme would allocate all the power to the strong user and zero power to the blocked one, which leads to improving the sum-rate (because all the power would be assigned to the strongest channel), but with a poor fairness. While in the proposed scheme (Co-NOMA with hybrid VLC/RF), the blocked user can be reached by the hybrid VLC/RF link, and the proposed scheme would divide the power between the strong user and the weak user so as to maximize the weighted sum-rate, 


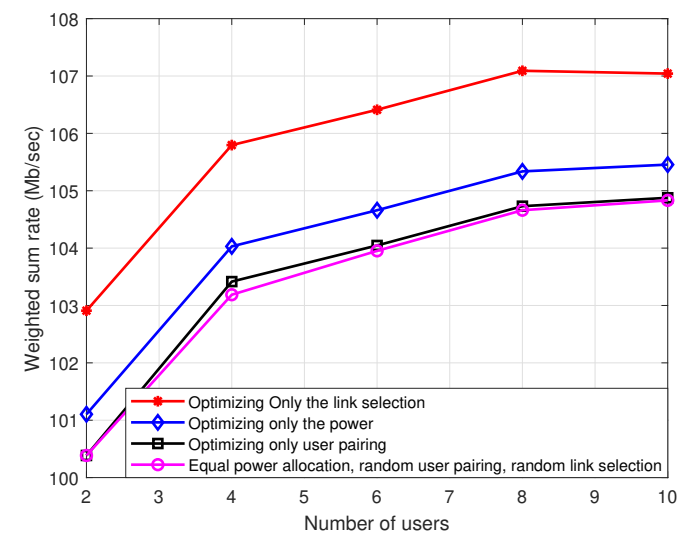

Fig. 10. Weighted sum rate versus number of users.

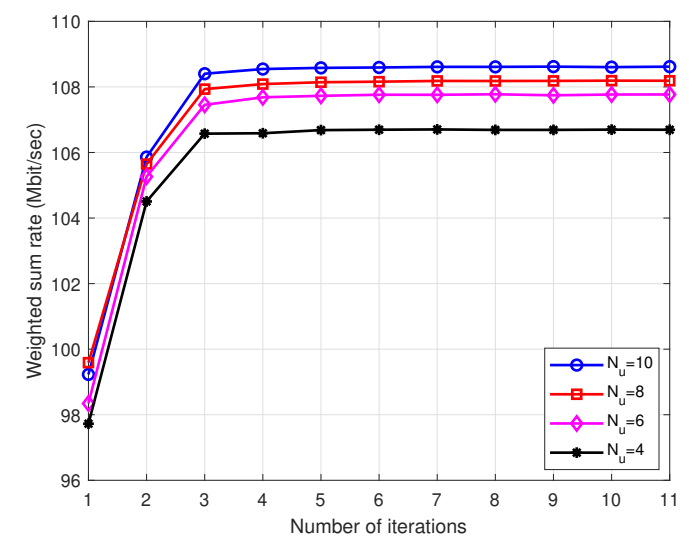

Fig. 11. Convergence of the proposed algorithm (Algorithm 2).

thereby enhancing fairness. Having said that, we wish to reemphasize that the proposed scheme still performs better than NOMA in terms of sum-rate, mainly because some of the weak users have non-zero, yet poor, channels. Therefore, the hybrid VLC/RF links would provide better service than the direct VLC link, especially that the proposed scheme enables the weak user to select the best link, while NOMA scheme would exclusively serve the weak users through the VLC link.

Fig. 10 shows the performance of Co-NOMA if we optimize each parameter individually and ignore the others. Specifically, the figure shows the results of not optimizing all variables (i.e., equal power allocation with generating random feasible solutions for the link selection and user pairing problems), optimizing only the power, optimizing only the user pairing, and optimizing only the link selection. The figure shows that the link selection problem has the largest effect on the weighted sum-rate, because by selecting the proper link for each weak user, we can reach the blocked and the out-ofcoverage users.

Fig. 11 shows the convergence of Algorithm 2 by plotting the weighted sum rate versus the number of iterations with different number of users. In each iteration, we implement the power allocation (Algorithm 1), the link selection, and the user pairing solutions, and then calculate the resulted weighted sum rate. Fig. 11 shows that the proposed overall algorithm converges within few iterations. It can also be seen that the number of required iterations for convergence does not significantly depend on the number of users in the system, which further highlights the numerical convergence speed of our proposed algorithm.

\section{CONCLUSION AND FUtURE WORK}

VLC is expected to be one of the candidate technologies in meeting the targeted requirements of next generation wireless communication networks. This paper introduces a novel cooperative scheme among users for extending coverage, improving sum-rate, and maximizing fairness in VLC systems. This cooperation is based on Co-NOMA, which can provide another chance for poorly serviced users to be served through a hybrid dual-hop VLC/RF link with the help of the well serviced users. The paper formulates an optimization problem that maximizes the weighted sum-rate by jointly allocating the power for users, pairing the users, and selecting the links for the weak users. An efficient, heuristic, iterative solution is proposed and compared with the exhaustive search approach, a simpler baseline solution, and with the traditional NOMA scheme. Simulation results show that the proposed system model and algorithms provide a significant improvement in terms of fairness and on top of that a slight improvement in terms of sum-rate compared to the traditional optimized NOMA scheme.

As a future work, we recommend to extend this cooperation scheme to coordinated multi-point VLC systems and in multi-cell VLC systems. Thanks to the fact that the RF does not interfere with VLC links, this cooperation scheme allows to mitigate the interference at edge users by serving them through center users using hybrid VLC/RL links. The cooperation among users, using RF links, is also helpful in mutli-user dynamic systems. We also recommend to extend the proposed scheme to multi-cell mobile users, where the handover should be considered and the channel estimation should be investigated. For multi-point coordinated transmission systems, we recommend to compare the proposed CoNOMA scheme with Co-OMA and cooperative rate-splitting schemes and show which one performs better than the others for various system requirements.

\section{APPENDIX A \\ PROOF OF PROPOSITION 1}

By writing the objective function in (20) as

$$
\begin{aligned}
R_{i, j}=w_{j}^{(s)} \frac{B_{v}}{2 K} \log _{2} & \left(1+\Psi_{j}^{(s)} P_{j}^{(s)}\right) \\
& +w_{i}^{(w)} \frac{B_{v}}{2 K} \log _{2}\left(\frac{\Psi_{j}^{(s)} q_{i, j}+1}{\Psi_{j}^{(s)} P_{j}^{(s)}+1}\right),
\end{aligned}
$$

and by differentiating the function $R_{i, j}$ with respect to $P_{j}^{(s)}$, we obtain

$$
\frac{d R_{i, j}}{d P_{j}^{(s)}}=\frac{\Psi_{j}^{(s)} B_{v}\left(w_{j}^{(s)}-w_{i}^{(w)}\right)}{2 K P_{j}^{(s)}} .
$$


From (34), we can see that the objective function is an increasing function of $P_{j}^{(s)}$ if $w_{j}^{(s)}>w_{i}^{(w)}$, a decreasing function if $w_{j}^{(s)}<w_{i}^{(w)}$, and constant if $w_{j}^{(s)}=w_{i}^{(w)}$. This means that the sum-rate function is a constant function of $P_{j}^{(s)}$ and modifying the values of $w_{i}^{(w)}$ and $w_{j}^{(s)}$ just affects the weighted sum-rate but not the sum-rate itself. Hence, the maximum fairness can be implemented without any degradation in the sum-rate with setting $w_{i}^{(w)}=w_{j}^{(s)}$ and having that the rate of the strong user equal to the rate of the weak user. To achieve that, $P_{j}^{(s)}$ must be selected to satisfy the following relation

$$
\frac{B_{v}}{2 K} \log _{2}\left(1+\Psi_{j}^{(s)} P_{j}^{(s)}\right)=\frac{B_{v}}{2 K} \log _{2}\left(\frac{\Psi_{j}^{(s)} q_{i, j}+1}{\Psi_{j}^{(s)} P_{j}^{(s)}+1}\right) .
$$

Solving (35), we obtain that $P_{j}^{(s)}=\eta_{i, j, 1}$, where $\eta_{i, j, 1}$ is given by

$$
\eta_{i, j, 1}=\frac{-1+\sqrt{1+q_{i, j} \Psi_{j}^{(s)}}}{\Psi_{j}^{(s)}}, \quad P_{i}^{(w)}=q_{i, j}-\eta_{i, j, 1} .
$$

Clearly, (36) achieves constraint (21c), whereas constraint (21d) needs to be investigated. Constraint (21d) can be expressed as $P_{j}^{(s)} \leq \eta_{i, j, 2}$. To guarantee achieving constraint (21d), the optimal $P_{j}^{(s)}$ must be $P_{j}^{(s)}=\min \left(\eta_{i, j, 1}, \eta_{i, j, 2}\right)$.

\section{APPENDIX B}

\section{ProOF OF Proposition 2}

It can be seen that the Hessian matrix of the objective function in (27) is negative definite whether $F_{j}^{(s)}\left(q_{i, j}\right)$ and $F_{i}^{(w)}\left(q_{i, j}\right)$ are given by (28) and (29), or given by (30) and (31), respectively. In addition, the constraints (27) are linear, which shows that problem (27) is convex. To find an optimal closed-form solution for $q_{i, j} \forall i, j$, write first the Lagrangian dual function:

$$
\begin{aligned}
& \zeta=-\sum_{i=1}^{K} \sum_{j=1}^{K} x_{i} z_{i, j}\left(w_{j}^{(s)} F_{j}^{(s)}\left(q_{i, j}\right)+w_{i}^{(w)} F_{i}^{(w)}\left(q_{i, j}\right)\right) \\
&-\sum_{i=1}^{K} \sum_{j=1}^{K}\left(1-x_{i}\right) z_{i, j} w_{i}^{(w)} \frac{B_{v}}{2 K} \log _{2}\left(1+\Omega_{i, j} \Psi_{j}^{(s)}\right) \\
&- \sum_{i=1}^{K} \sum_{j=1}^{K}\left(1-x_{i}\right) z_{i, j} w_{i}^{(w)} \frac{B_{v}}{2 K} \log _{2}\left(\frac{q_{i, j} \Psi_{i}^{(w)}+1}{\Omega_{i, j} \Psi_{i}^{(w)}+1}\right)+ \\
& \lambda\left(\sum_{i=1}^{K} \sum_{j=1}^{K} q_{i, j}-P_{\max }\right)+\sum_{i=1}^{K} \sum_{j=1}^{K} \mu_{i, j} q_{i, j}
\end{aligned}
$$

where $\lambda$ is the dual variable associated with the sumpower constraint. Based on the first-order Karush-KuhnTucker (KKT) conditions [47], we have

$$
\frac{\partial \zeta}{\partial q_{i, j}}=0, \forall i, j \text {. }
$$

We have three cases. In the first case, if $z_{i, j}=0$ (i.e., users $i$ and $j$ are not paired), $q_{i, j}=0$ whether $x_{i}=1$ or $x_{i}=0$.
The second case occurs when $z_{i, j}=1$ and $x_{i}=1$, and so we can reformulate (38) as

$$
\begin{aligned}
\frac{\partial}{\partial q_{i, j}}\left[-w_{j}^{(s)} F_{j}^{(s)}\right. & \left(q_{i, j}\right)-w_{i}^{(w)} F_{i}^{(w)}\left(q_{i, j}\right) \\
& \left.+\lambda\left(q_{i, j}-P_{\max }\right)+\mu_{i, j} q_{i, j}\right]=0 .
\end{aligned}
$$

If $F_{j}^{(s)}$ and $F_{i}^{(w)}$ are given by (28) and (29), respectively, (39) can be given by

$$
\begin{aligned}
\frac{\partial}{\partial q_{i, j}}\left[-2 w_{j}^{(w)} \frac{B_{v}}{2 K} \log _{2}\left(\sqrt{\Psi_{j}^{(s)} q_{i, j}+1}\right)\right. \\
\left.+\lambda\left(q_{i, j}-P_{\max }\right)+\mu_{i, j} q_{i, j}\right]=0,
\end{aligned}
$$

where $w_{i}^{(w)}=w_{j}^{(s)}$ because $F_{j}^{(s)}=F_{i}^{(w)}$ in this case. On the other hand, if $F_{j}^{(s)}$ and $F_{i}^{(w)}$ are given by (30) and (31), respectively, (39) can be given by

$$
\begin{aligned}
& \frac{\partial}{\partial q_{i, j}}\left[-w_{j}^{(w)} \frac{B_{v}}{2 K} \log _{2}\left(\frac{\Psi_{j}^{(s)} q_{i, j}+1}{A}\right)\right. \\
& \left.-w_{i}^{(w)} R_{i, j}^{R F}(\mathbf{x})+\lambda\left(q_{i, j}-P_{\max }\right)+\mu_{i, j} q_{i, j}\right]=0,
\end{aligned}
$$

Solving (40) or (41), we obtain the same expression for $q_{i, j}$, which is given by

$$
q_{i, j}=\left[\frac{w_{j}^{(w)} B_{v}}{2 K \lambda}-\frac{1}{\Psi_{j}^{(s)}}\right]^{+} .
$$

Finally, in the third case, if we have $z_{i, j}=1$ and $x_{i}=0$ (the weak user is served through the direct VLC link), which allows to rewrite the first-order condition (38) as follows:

$$
\begin{aligned}
\frac{\partial}{\partial q_{i, j}}\left[-w_{i}^{(w)} \frac{B_{v}}{2 K}\right. & \log _{2}\left(\frac{q_{i, j} \Psi_{i}+1}{\Omega_{i, j} \Psi_{i}^{(w)}+1}\right) \\
& \left.+\lambda\left(q_{i, j}-P_{\max }\right)+\mu_{i, j} q_{i, j}\right]=0
\end{aligned}
$$

Solving (43), we get

$$
q_{i, j}=\left[\frac{w_{i}^{(w)} B_{v}}{2 K \lambda}-\frac{1}{\Psi_{j}^{(s)}}\right]^{+} .
$$

\section{ACKNOWLEDGMENT}

The authors acknowledge funding from the Research and Development (R\&D) Program (Research Pooling Initiative), Ministry of Education, Riyadh, Saudi Arabia.

\section{REFERENCES}

[1] B. Schrenk, M. Hofer, F. Laudenbach, H. Hübel, and T. Zemen, "Visible-light multi-Gb/s transmission based on resonant cavity LED with optical energy feed," IEEE J. Sel. Areas Commun., vol. 36, no. 1, pp. 175-184, Jan. 2018.

[2] D. Tsonev, S. Videv, and H. Haas, "Towards a $100 \mathrm{~Gb} / \mathrm{s}$ visible light wireless access network," Opt. Express, vol. 23, no. 2, pp. 1627-1637, Jan. 2015.

[3] L. Yin and H. Haas, "Coverage analysis of multiuser visible light communication networks," IEEE Trans. Wireless Commun, vol. 17, no. 3, pp. 1630-1643, Mar. 2018.

[4] M. Obeed, A. M. Salhab, M.-S. Alouini, and S. A. Zummo, "On optimizing VLC networks for downlink multi-user transmission: A survey," IEEE Commun. Surveys Tuts., vol. 21, no. 3, pp. 2947-2976, thirdquarter 2019. 
[5] Y. Liu, Z. Ding, M. Elkashlan, and H. V. Poor, "Cooperative nonorthogonal multiple access with simultaneous wireless information and power transfer," IEEE J. Sel. Areas Commun., vol. 34, no. 4, pp. 938953, Apr. 2016.

[6] Z. Wang, D. Tsonev, S. Videv, and H. Haas, "On the design of a solarpanel receiver for optical wireless communications with simultaneous energy harvesting," IEEE J. Sel. Areas Commun., vol. 33, no. 8, pp. 1612-1623, Aug. 2015.

[7] M. Obeed, H. Dahrouj, A. M. Salhab, S. A. Zummo, and M.-S. Alouini, "DC-Bias and power allocation in cooperative VLC networks for joint information and energy transfer," IEEE Trans. Wireless Commun., vol. 18 , no. 12 , pp. 5486-5499, Dec. 2019.

[8] X. Li, R. Zhang, and L. Hanzo, "Cooperative load balancing in hybrid visible light communications and WiFi," IEEE Trans. Commun., vol. 63, no. 4, pp. 1319-1329, Apr. 2015.

[9] Y. Wang and H. Haas, "Dynamic load balancing with handover in hybrid Li-Fi and Wi-Fi networks," J. Lightw. Technol., vol. 33, no. 22, pp. 4671-4682, Nov. 2015.

[10] Y. Wang, D. A. Basnayaka, X. Wu, and H. Haas, "Optimization of load balancing in hybrid LiFi/RF networks," IEEE Trans. Commun., vol. 65 , no. 4, pp. 1708-1720, Apr. 2017.

[11] X. Wu and H. Haas, "Access point assignment in hybrid LiFi and WiFi networks in consideration of LiFi channel blockage," in 18th Int. Workshop Signal Processing Advances in Wireless Commun. (SPAWC). IEEE, July 2017, pp. 1-5.

[12] M. Obeed, A. M. Salhab, S. A. Zummo, and M.-S. Alouini, "Joint optimization of power allocation and load balancing for hybrid VLC/RF networks," J. Opt. Commun. Netw., vol. 10, no. 5, pp. 553-562, May 2018.

[13] —, "Joint load balancing and power allocation for hybrid VLC/RF networks," in Proc. Global Commun. Conf. (Globecom). Singapore: IEEE, 2017

[14] R. Zhang, H. Claussen, H. Haas, and L. Hanzo, "Energy efficient visible light communications relying on amorphous cells," IEEE J. Sel. Areas Commun., vol. 34, no. 4, pp. 894-906, 2016.

[15] M. Obeed, A. M. Salhab, S. A. Zummo, and M.-S. Alouini, "New algorithms for energy-efficient VLC networks with user-centric cell formation," IEEE Trans. Green Commun. and Netw., vol. 3, no. 1, pp. 108-121, Mar. 2019.

[16] C. Chen, D. Tsonev, and H. Haas, "Joint transmission in indoor visible light communication downlink cellular networks," in Proc. Global Commun. Conf. (Globecom). IEEE, 2013, pp. 1127-1132.

[17] C. Chen and H. Haas, "Performance evaluation of downlink cooperative multipoint joint transmission in LiFi systems," in Proc. Globecom Wkshps (GC Wkshps). IEEE, 2017, pp. 1-6.

[18] T. Rakia, H.-C. Yang, F. Gebali, and M.-S. Alouini, "Optimal design of dual-hop VLC/RF communication system with energy harvesting," IEEE Commun. Lett., vol. 20, no. 10, pp. 1979-1982, Oct. 2016.

[19] — - "Dual-hop VLC/RF transmission system with energy harvesting relay under delay constraint," in Globecom Workshops (GC Wkshps). IEEE, 2016, pp. 1-6.

[20] R. C. Kizilirmak, O. Narmanlioglu, and M. Uysal, "Relay-assisted OFDM-based visible light communications," IEEE Trans. Commun., vol. 63 , no. 10 , pp. 3765-3778, Oct. 2015.

[21] P. D. Diamantoulakis, G. K. Karagiannidis, and Z. Ding, "Simultaneous lightwave information and power transfer (SLIPT)," IEEE Trans. Green Commun. and Netw., vol. 2, no. 3, pp. 764-773, Sep. 2018.

[22] G. Pan, P. D. Diamantoulakis, Z. Ma, Z. Ding, and G. K. Karagiannidis, "Simultaneous lightwave information and power transfer: Policies, techniques, and future directions," IEEE Access, vol. 7, pp. 28250 $28257,2019$.

[23] Z. Ding, X. Lei, G. K. Karagiannidis, R. Schober, J. Yuan, and V. K. Bhargava, "A survey on non-orthogonal multiple access for $5 \mathrm{G}$ networks: Research challenges and future trends," IEEE J. Sel. Areas Commun., vol. 35, no. 10, pp. 2181-2195, Oct. 2017.

[24] Y. Liu, Z. Qin, M. Elkashlan, Z. Ding, A. Nallanathan, and L. Hanzo, "Nonorthogonal multiple access for 5G and beyond," Proc. IEEE, vol. 105, no. 12, pp. 2347-2381, Dec. 2017.

[25] R. C. Kizilirmak, C. R. Rowell, and M. Uysal, "Non-orthogonal multiple access (NOMA) for indoor visible light communications," in 4th Int. Workshop Opt. Wireless Commun. (IWOW). IEEE, 2015, pp. 98-101.

[26] L. Yin, W. O. Popoola, X. Wu, and H. Haas, "Performance evaluation of non-orthogonal multiple access in visible light communication," IEEE Trans. Commun., vol. 64, no. 12, pp. 5162-5175, Dec. 2016.

[27] Y. Yapici and I. Guvenc, "Non-orthogonal multiple access for mobile VLC networks with random receiver orientation," arXiv preprint arXiv:1801.04888, 2018.
[28] H. Marshoud, V. M. Kapinas, G. K. Karagiannidis, and S. Muhaidat, "Non-orthogonal multiple access for visible light communications," IEEE Photon. Technol. Lett, vol. 28, no. 1, pp. 51-54, Jan. 2016.

[29] X. Zhang, Q. Gao, C. Gong, and Z. Xu, "User grouping and power allocation for NOMA visible light communication multi-cell networks," IEEE Commun. Lett., vol. 21, no. 4, pp. 777-780, Apr. 2017.

[30] S. Ma, Y. He, H. Li, S. Lu, F. Zhang, and S. Li, "Optimal power allocation for mobile users in non-orthogonal multiple access visible light communication networks," IEEE Trans. Commun., vol. 67, no. 3, pp. 2233-2244, Mar. 2018

[31] S. Ma, H. Li, Y. He, R. Yang, S. Lu, W. Cao, and S. Li, "Capacity bounds and interference management for interference channel in visible light communication networks," IEEE Trans. Wireless Commun., vol. 18, no. 1, pp. 182-193, Jan. 2018.

[32] X. Zhou, S. Li, H. Zhang, Y. Wen, Y. Han, and D. Yuan, "Cooperative NOMA based VLC/RF system with simultaneous wireless information and power transfer," in 2018 IEEE/CIC International Conference on Communications in China (ICCC). IEEE, Aug. 2018.

[33] Y. Xiao, P. D. Diamantoulakis, Z. Fang, Z. Ma, L. Hao, and G. K. Karagiannidis, "Hybrid lightwave/RF cooperative NOMA networks," IEEE Trans. Wireless Commun., Feb. 2019.

[34] W. Yu, T. Kwon, and C. Shin, "Multicell coordination via joint scheduling, beamforming and power spectrum adaptation," in 2011 Proceedings IEEE INFOCOM. IEEE, Apr. 2011.

[35] J. M. Kahn and J. R. Barry, "Wireless infrared communications," Proc. IEEE, vol. 85, no. 2, pp. 265-298, 1997.

[36] E. Perahia and R. Stacey, Next Generation Wireless LANs. Cambridge University Press, 2013

[37] Q. Gao, C. Gong, and Z. Xu, "Joint transceiver and offset design for visible light communications with input-dependent shot noise," IEEE Trans. Wireless Commun., vol. 16, no. 5, pp. 2736-2747, May 2017.

[38] A. Chaaban, Z. Rezki, and M.-S. Alouini, "On the capacity of the intensity-modulation direct-detection optical broadcast channel," IEEE Trans. Wireless Commun., vol. 15, no. 5, pp. 3114-3130, May 2016.

[39] C. Li, W. Jia, Q. Tao, and M. Sun, "Solar cell phone charger performance in indoor environment," in IEEE 37th Annu. Northeast Bioengineering Conference (NEBEC). IEEE, 2011, pp. 1-2.

[40] J. Zhu, J. Wang, Y. Huang, S. He, X. You, and L. Yang, "On optimal power allocation for downlink non-orthogonal multiple access systems," IEEE J. Sel. Areas Commun., pp. 1-1, Dec. 2017.

[41] H. W. Kuhn, "The hungarian method for the assignment problem," Naval Research Logistics, vol. 52, no. 1, pp. 7-21, Feb. 2005.

[42] G. A. Mills-Tettey, A. Stentz, and M. B. Dias, "The dynamic hungarian algorithm for the assignment problem with changing costs," 2007.

[43] A. Douik, H. Dahrouj, T. Y. Al-Naffouri, and M.-S. Alouini, "A tutorial on clique problems in communications and signal processing," Proceedings of the IEEE, vol. 108, no. 4, pp. 583-608, 2020.

[44] L. Costa and P. Oliveira, "Evolutionary algorithms approach to the solution of mixed integer non-linear programming problems," Computers \& Chemical Engineering, vol. 25, no. 2-3, pp. 257-266, 2001.

[45] S. Burer and A. N. Letchford, "Non-convex mixed-integer nonlinear programming: A survey," Surveys in Operations Research and Management Science, vol. 17, no. 2, pp. 97-106, 2012.

[46] A. Grinman, "The hungarian algorithm for weighted bipartite graphs," Massachusetts Institute of Technology, 2015.

[47] S. Boyd and L. Vandenberghe, Convex Optimization. Cambridge University Press, 2004 


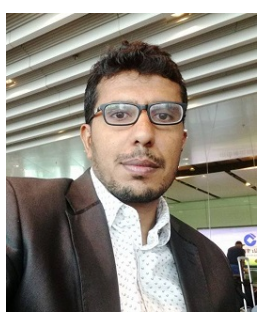

Mohanad Obeed received the B.Eng. degree in computer and communication engineering from Taiz University, Taiz, Yemen, in 2008, the M.Sc. and the Ph.D. degree in electrical engineering from King Fahd University of Petroleum and Minerals (KFUPM), Dhahran, Saudi Arabia, in 2016 and 2019, respectively. From July 2017 to July 2019, he was a visiting student at King Abdullah University of Science and Technology (KAUST) under the supervision of Mohamed-Slim Alouini. He is currently a Postdoctoral Research Fellow with the School of Engineering at the University of British Columbia, Canada. His research interests include visible light communications, cooperative networks, resource allocation, convex optimization, physical layer security, and energy harvesting.

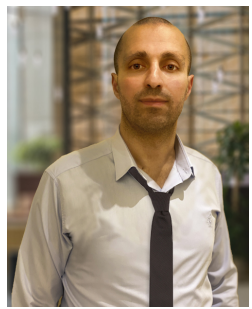

Hayssam Dahrouj (S'02, M'11, SM'15) received his B.E. degree (with high distinction) in computer and communications engineering from the American University of Beirut (AUB), Lebanon, in 2005, and his Ph.D. degree in electrical and computer engineering from the University of Toronto (UofT), Canada, in 2010. In July 2020, he joined the Center of Excellence for NEOM Research at King Abdullah University of Science and Technology (KAUST) as a senior research scientist. From June 2015 to June 2020, he was with the Department of Electrical and Computer Engineering at Effat University as an assistant professor, and as a visiting scholar at the Computer, Electrical and Mathematical Sciences and Engineering (CEMSE) division at KAUST, where he also was a research associate between April 2014 and May 2015. Prior to joining KAUST, he was an industrial postdoctoral fellow at UofT, in collaboration with BLiNQ Networks Inc., Kanata, Canada, where he worked on developing practical solutions for the design of non-line-of sight wireless backhaul networks. His contributions to the field led to five patents. During his doctoral studies at UofT, he pioneered the idea of coordinated beamforming as a means of minimizing intercell interference across multiple base stations. The journal paper on this subject was ranked second in the 2013 IEEE Marconi paper awards in wireless communications. Dr. Dahrouj is the recipient of both the faculty award of excellence in research, and the faculty award of excellence in teaching (at the university level) in May 2017. He is a senior member of the IEEE, an associate editor of the Frontiers in Communications and Networks, and a lead-guest editor of the Frontiers special issue on Resource Allocation in Cloud-Radio Access Networks and Fog-Radio Access Networks for B5G Systems. His main research interests include 6G wireless systems, cloud- and fog-radio access networks, cross-layer optimization, cooperative networks, convex optimization, distributed algorithms, and optical communications.

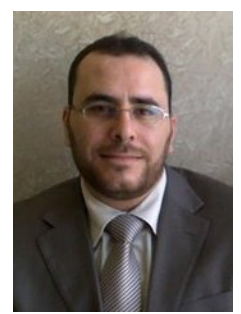

Anas M. Salhab (S'11-M'14-SM'17) received the B.Sc. degree in electrical engineering from Palestine Polytechnic University, Hebron, Palestine, in 2004, the M.Sc. degree in electrical engineering from Jordan University of Science and Technology, Irbid, Jordan, in 2007, and the Ph.D. degree from King Fahd University of Petroleum and Minerals (KFUPM), Dhahran, Saudi Arabia, in 2013. From 2013 to 2014, he was a Postdoctoral Fellow with the Electrical Engineering Department, KFUPM. He is currently an Associate Professor and the Assistant Director of Science \& Technology Unit with the Deanship of Scientific Research, KFUPM. His research interest spans special topics in modeling and performance analysis of wireless communication systems, including cooperative relay networks, cognitive radio relay networks, free space optical networks, visible light communications, and co-channel interference. He was selected as an Exemplary Reviewer by the IEEE WIRELESS COMMUNICATIONS LETTERS for his reviewing service in 2014. Recently, he has been nominated by ISI and SCOPUS among the first 10 faculty and researchers in KFUPM (2013-2018) for the "Excellence Award for Scientific Production (Engineering and Technology Field)" offered by the Saudi Digital Library, Ministry of Education, Saudi Arabia.

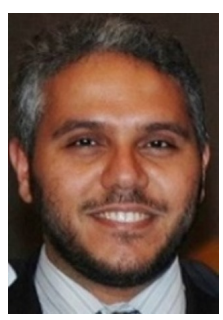

Salam A. Zummo (M'00-SM'08) received the B.Sc. and M.Sc. degrees in Electrical Engineering from King Fahd University of Petroleum \& Minerals (KFUPM), Dhahran, Saudi Arabia, in 1998 and 1999, respectively, and his $\mathrm{PhD}$ degree from the University of Michigan at Ann Arbor, USA, in June 2003. He is currently a Professor in the Electrical Engineering Department at KFUPM, and a Senior Member of the IEEE.

Prof. Zummo was awarded the Award for Outstanding Contribution to Education in 2016, and the KFUPM Excellence in Research Award for the years 2012 and 2019, and the KFUPM Best Research Project Award in 2016. In addition, he won the British Council/BAE Research Fellowship Awards in 2004 and 2006, and the Saudi Ambassador Award for early PhD completion in 2003. $\mathrm{He}$ has 15 issued US patents and more than 170 papers published in reputable journals and conference proceedings. His research interests are the area of wireless communications including MIMO systems, cooperative and multiuser diversity, relay networks, cognitive radio, physical layer security, visible light communications, error control coding, and interference modeling and analysis in wireless networks.

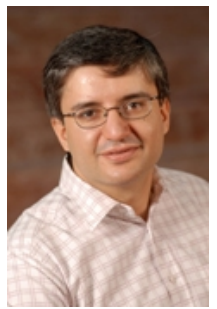

Mohamed-Slim Alouini (S'94, M'98, SM'03, F'09) was born in Tunis, Tunisia. He received the $\mathrm{Ph} . D$. degree in Electrical Engineering from the $\mathrm{Cal}-$ ifornia Institute of Technology (Caltech), Pasadena, CA, USA, in 1998. He served as a faculty member in the University of Minnesota, Minneapolis, MN USA, then in the Texas A\&M University at Qatar, Education City, Doha, Qatar before joining King Abdullah University of Science and Technology (KAUST), Thuwal, Makkah Province, Saudi Arabia as a Professor of Electrical Engineering in 2009. His current research interests include the modeling, design, and performance analysis of wireless communication systems. 